Pathway Search Result

\begin{tabular}{|c|c|c|c|}
\hline Code & Pathway name & Species & Number of proteins \\
\hline hsa04810 & Regulation of actin cytoskeleton & Homo sapiens (human) & 57 \\
\hline hsa04510 & Focal adhesion & Homo sapiens (human) & 52 \\
\hline hsa05200 & Pathways in cancer & Homo sapiens (human) & 51 \\
\hline hsa04144 & Endocytosis & Homo sapiens (human) & 43 \\
\hline hsa05016 & Huntington's disease & Homo sapiens (human) & 42 \\
\hline hsa05010 & Alzheimer's disease & Homo sapiens (human) & 41 \\
\hline hsa04010 & MAPK signaling pathway & Homo sapiens (human) & 40 \\
\hline hsa03040 & Spliceosome & Homo sapiens (human) & 40 \\
\hline hsa04142 & Lysosome & Homo sapiens (human) & 37 \\
\hline hsa04670 & Leukocyte transendothelial migration & Homo sapiens (human) & 36 \\
\hline hsa04062 & Chemokine signaling pathway & Homo sapiens (human) & 36 \\
\hline hsa04722 & Neurotrophin signaling pathway & Homo sapiens (human) & 34 \\
\hline hsa05322 & Systemic lupus erythematosus, & Homo sapiens (human) & 33 \\
\hline hsa04666 & Fc gamma R-mediated phagocytosis & Homo sapiens (human) & 32 \\
\hline hsa04310 & Wnt signaling pathway & Homo sapiens (human) & 31 \\
\hline hsa05012 & Parkinson's disease & Homo sapiens (human) & 31 \\
\hline hsa00190 & Oxidative phosphorylation & Homo sapiens (human) & 30 \\
\hline hsa04020 & Calcium signaling pathway & Homo sapiens (human) & 29 \\
\hline hsa03010 & Ribosome & Homo sapiens (human) & 28 \\
\hline hsa04910 & Insulin signaling pathway & Homo sapiens (human) & 28 \\
\hline hsa04650 & Natural killer cell mediated cytotoxicity & Homo sapiens (human) & 28 \\
\hline hsa04530 & Tight junction & Homo sapiens (human) & 26 \\
\hline hsa04916 & Melanogenesis & Homo sapiens (human) & 26 \\
\hline hsa00010 & Glycolysis / Gluconeogenesis & Homo sapiens (human) & 25 \\
\hline hsa05130 & Pathogenic Escherichia coli infection & Homo sapiens (human) & 25 \\
\hline hsa00230 & Purine metabolism & Homo sapiens (human) & 24 \\
\hline hsa04720 & Long-term potentiation & Homo sapiens (human) & 24 \\
\hline hsa03050 & Proteasome & Homo sapiens (human) & 24 \\
\hline hsa04270 & Vascular smooth muscle contraction & Homo sapiens (human) & 23 \\
\hline hsa04360 & Axon guidance & Homo sapiens (human) & 22 \\
\hline hsa04114 & Oocyte meiosis & Homo sapiens (human) & 22 \\
\hline hsa04912 & GnRH signaling pathway & Homo sapiens (human) & 21 \\
\hline hsa04540 & Gap junction & Homo sapiens (human) & 21 \\
\hline hsa04512 & ECM-receptor interaction & Homo sapiens (human) & 21 \\
\hline hsa04520 & Adherens junction & Homo sapiens (human) & 20 \\
\hline hsa04612 & Antigen processing and presentation & Homo sapiens (human) & 19 \\
\hline
\end{tabular}




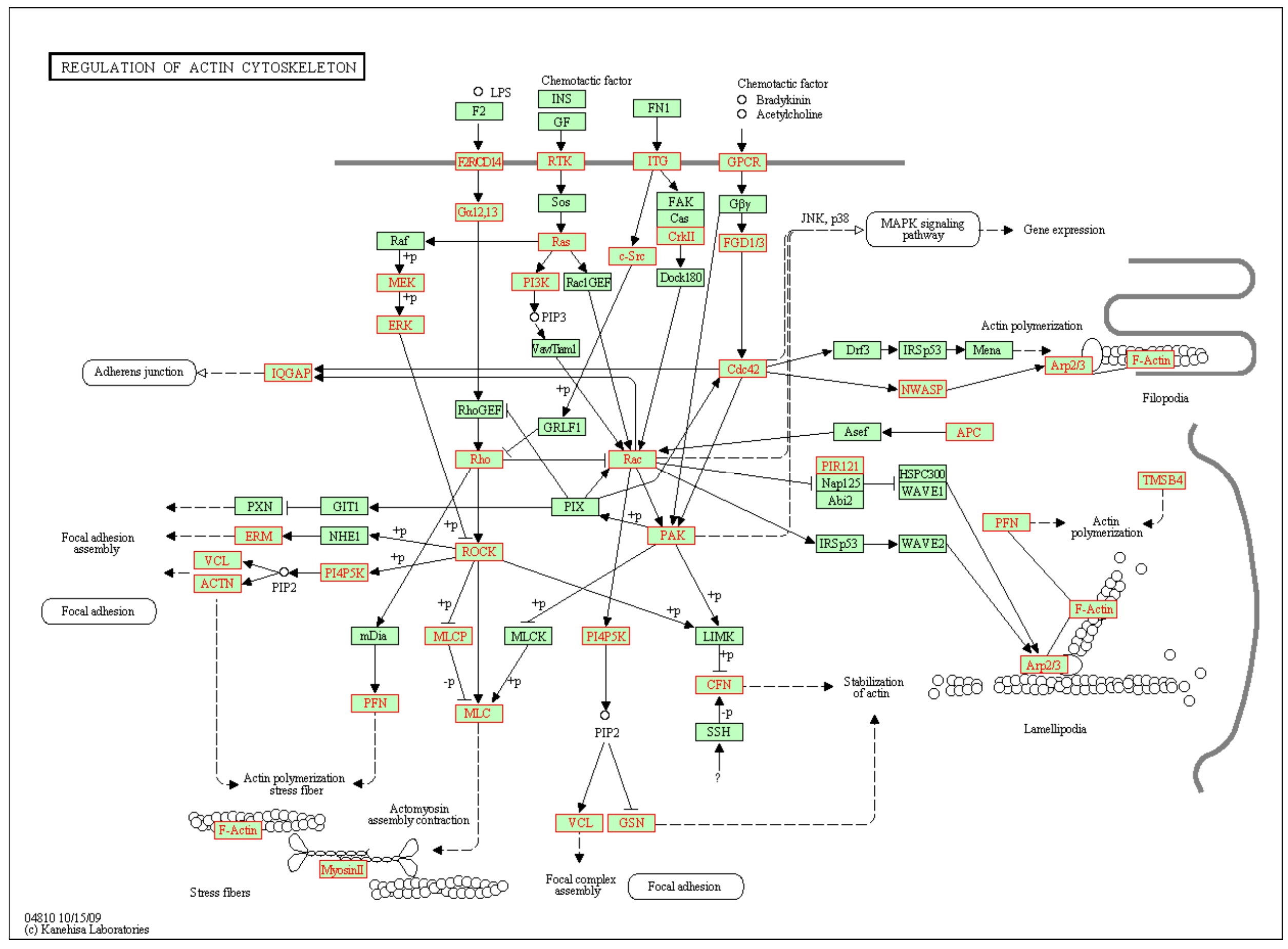




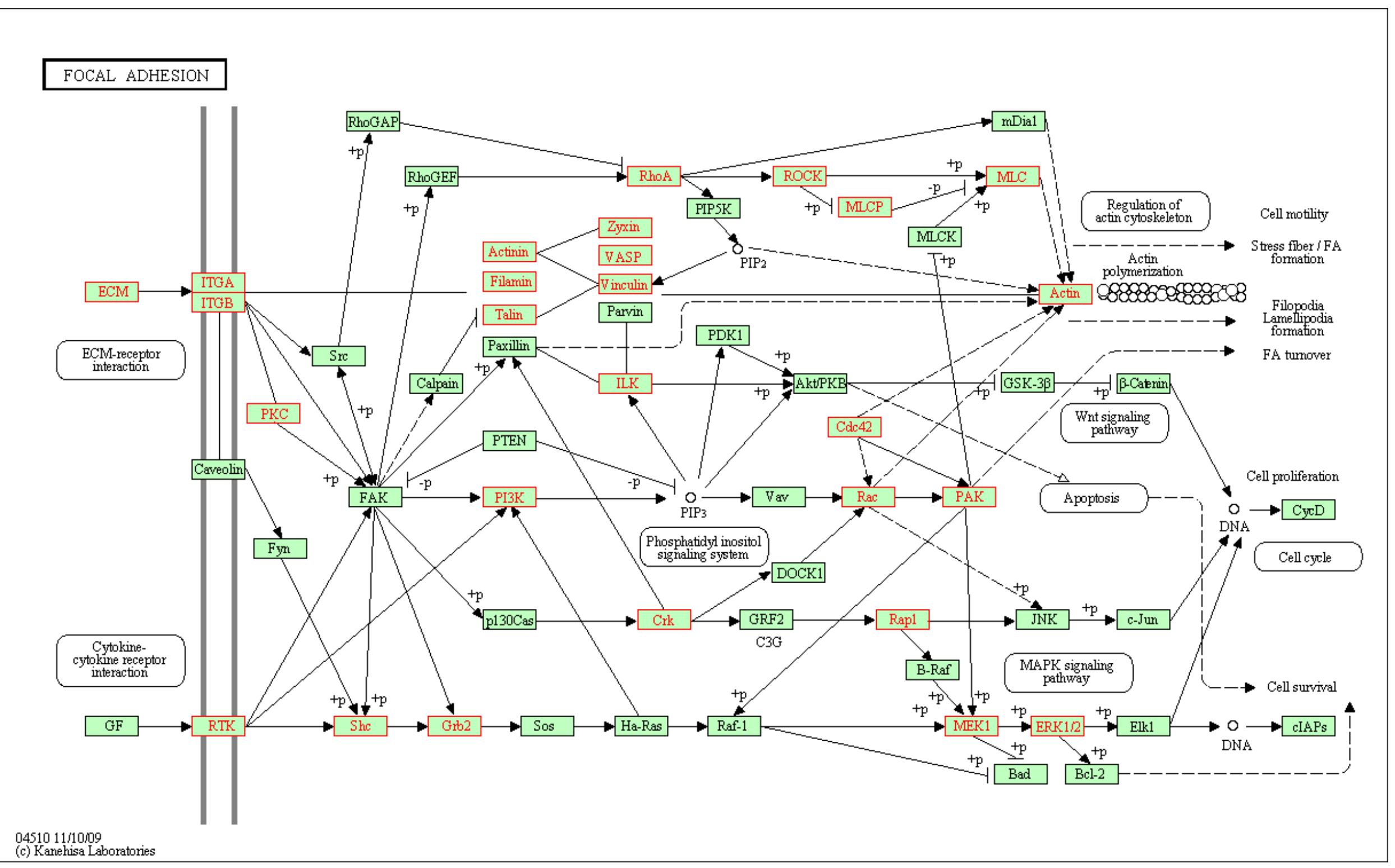




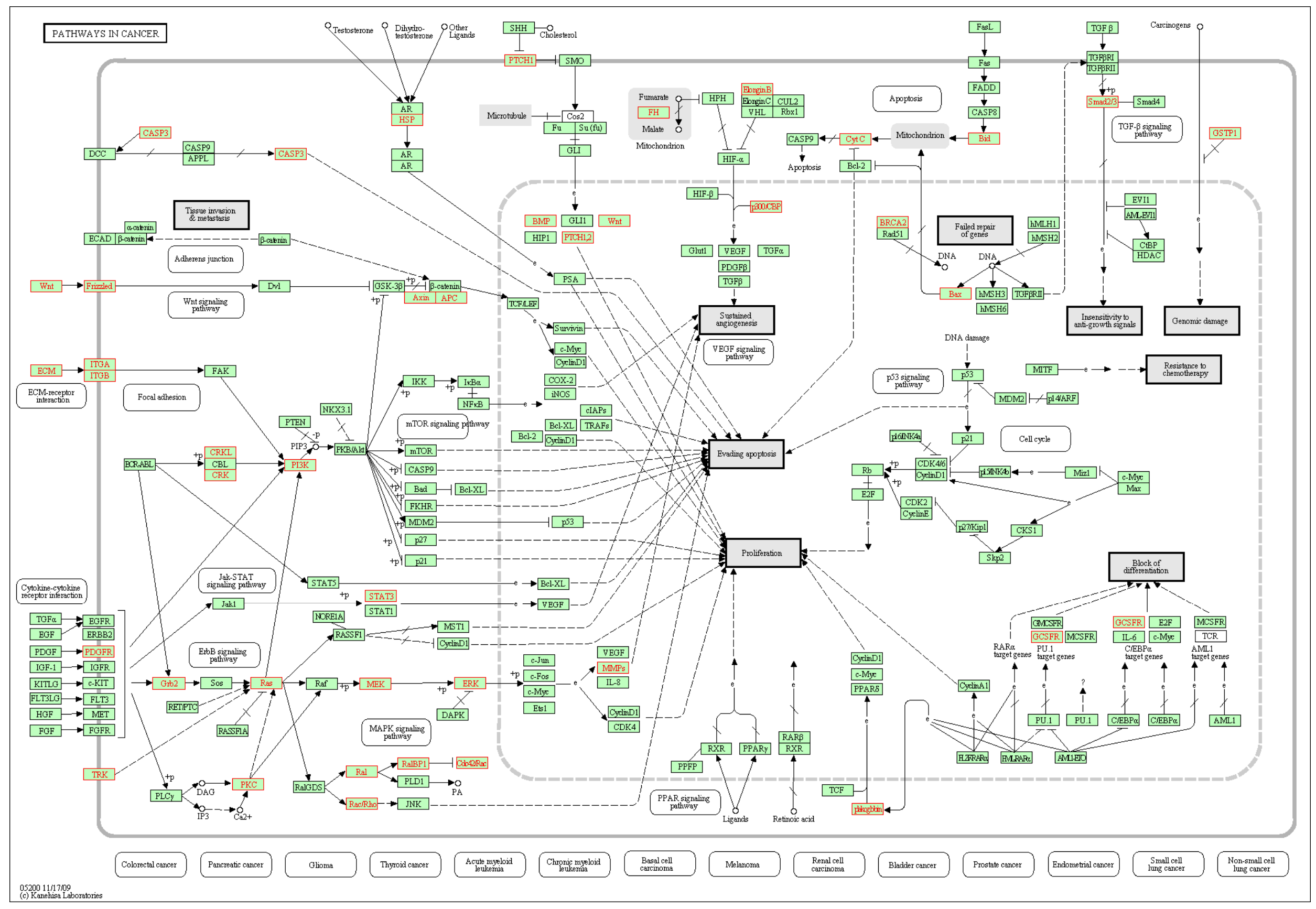




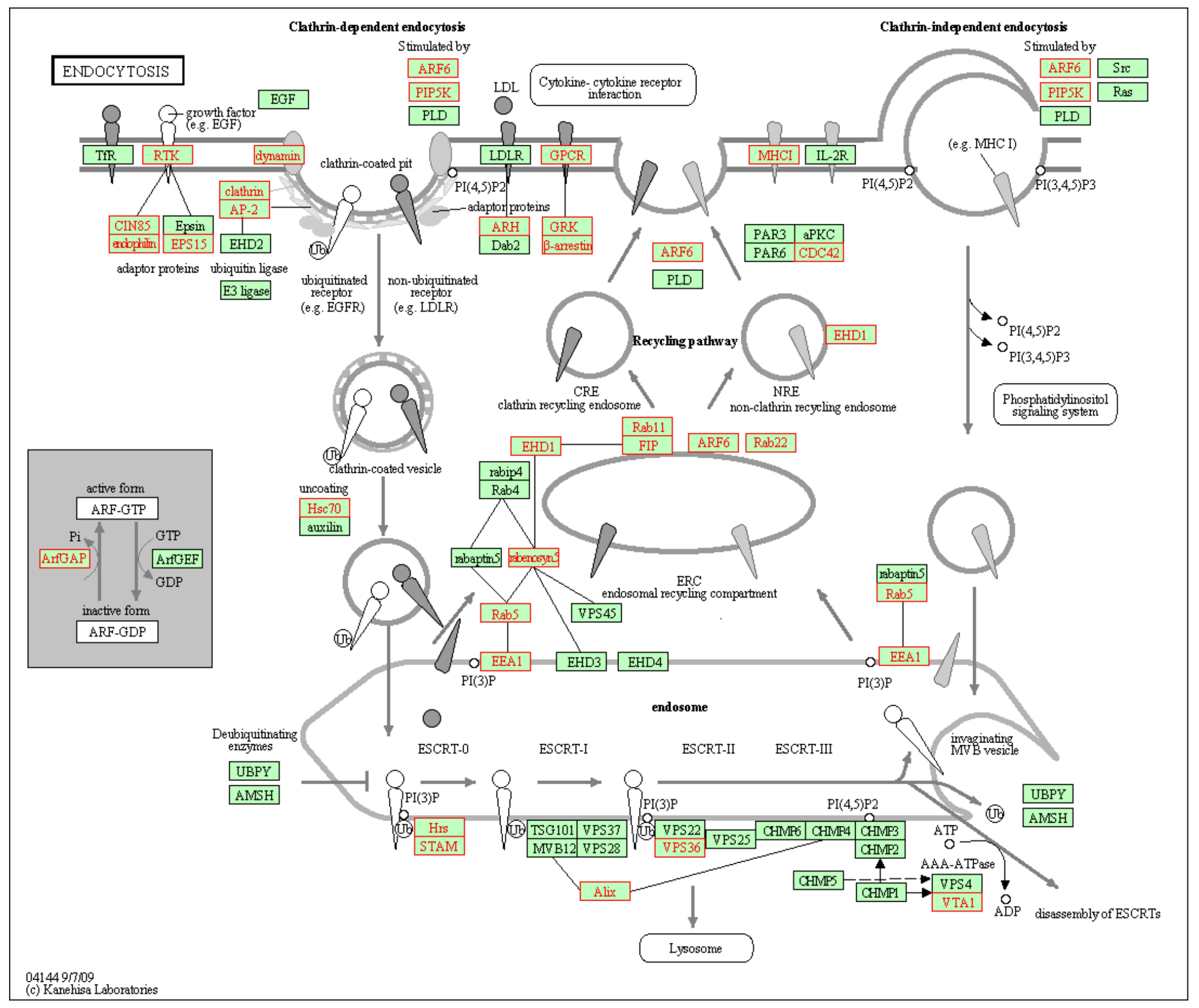




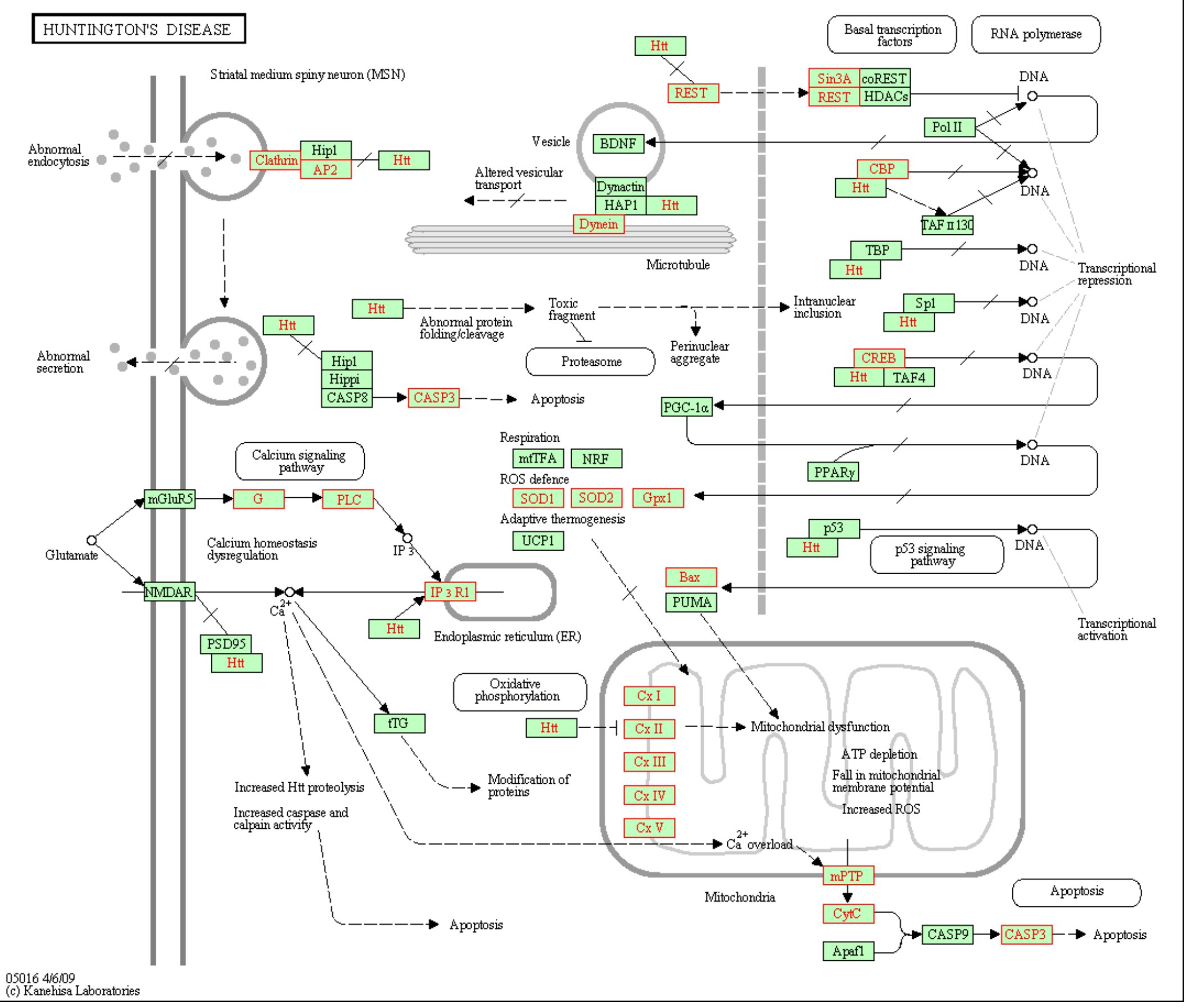




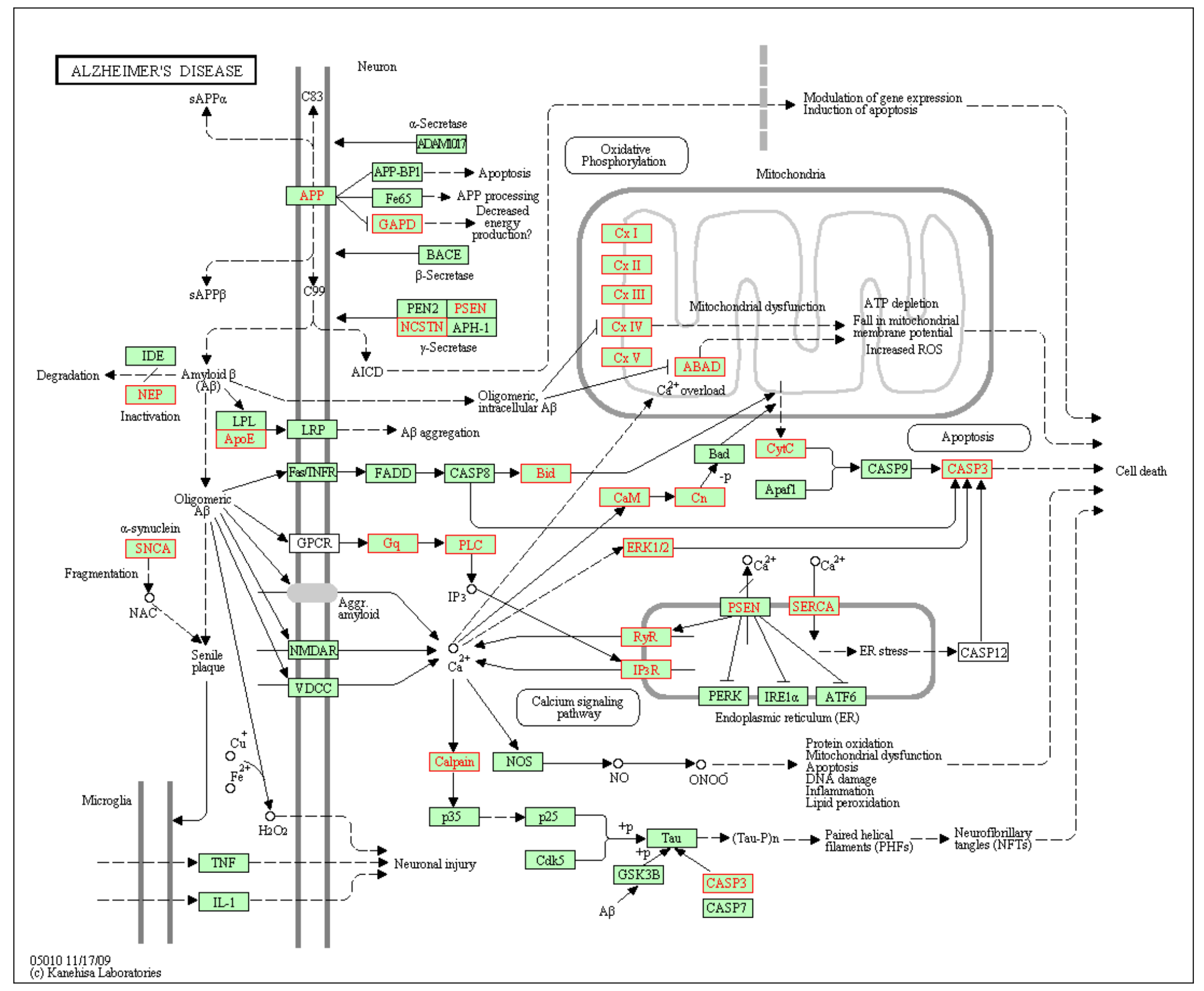




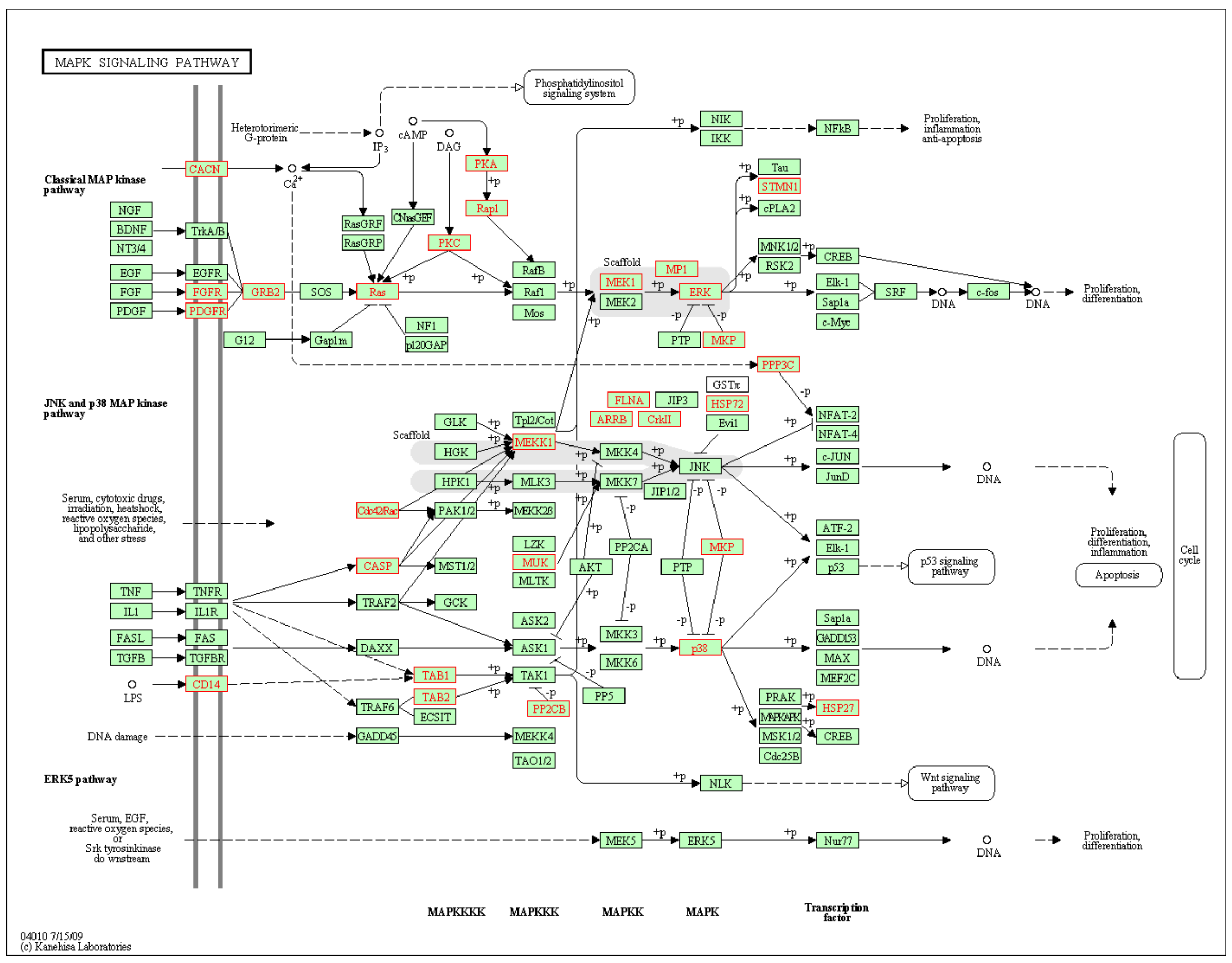




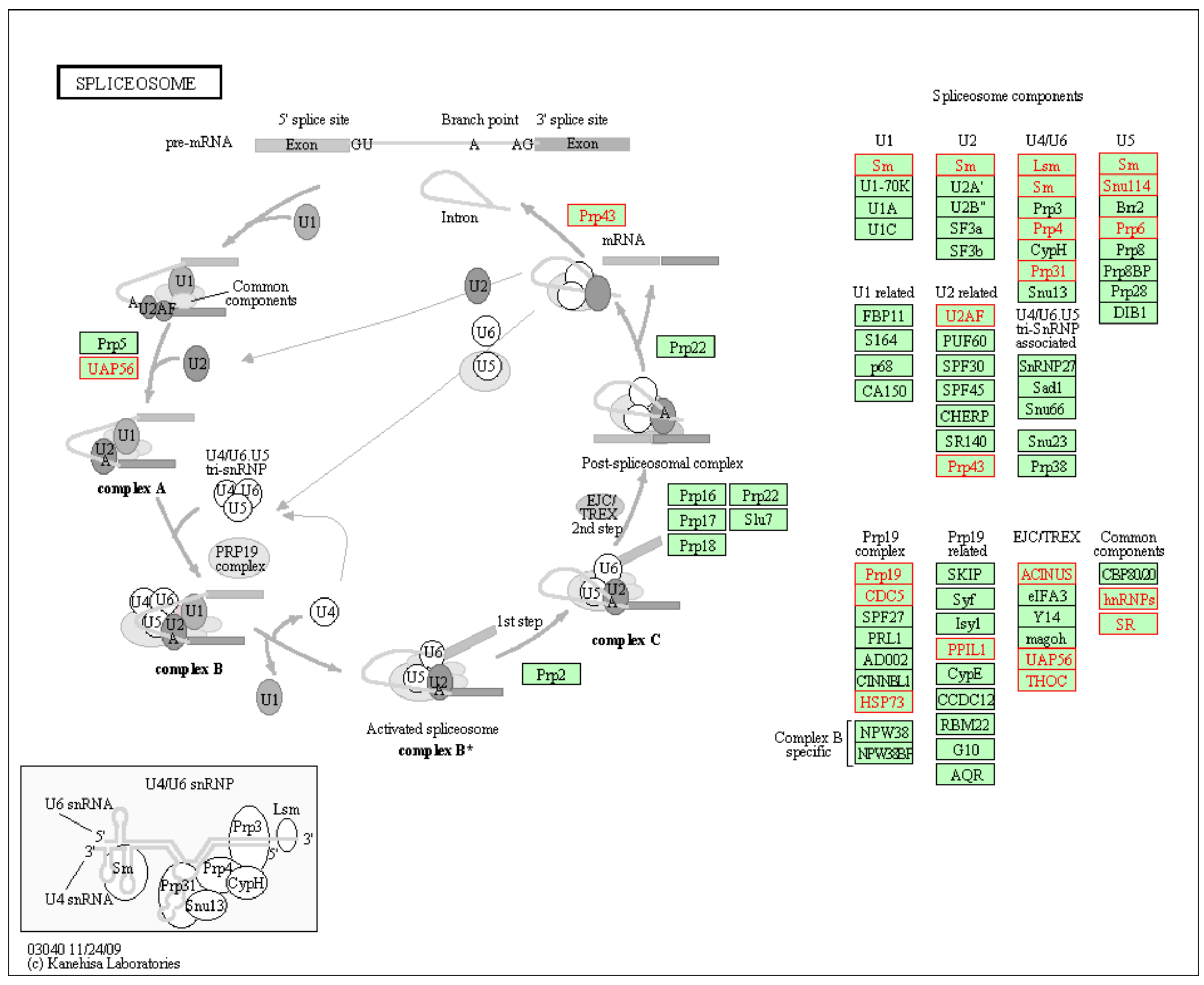




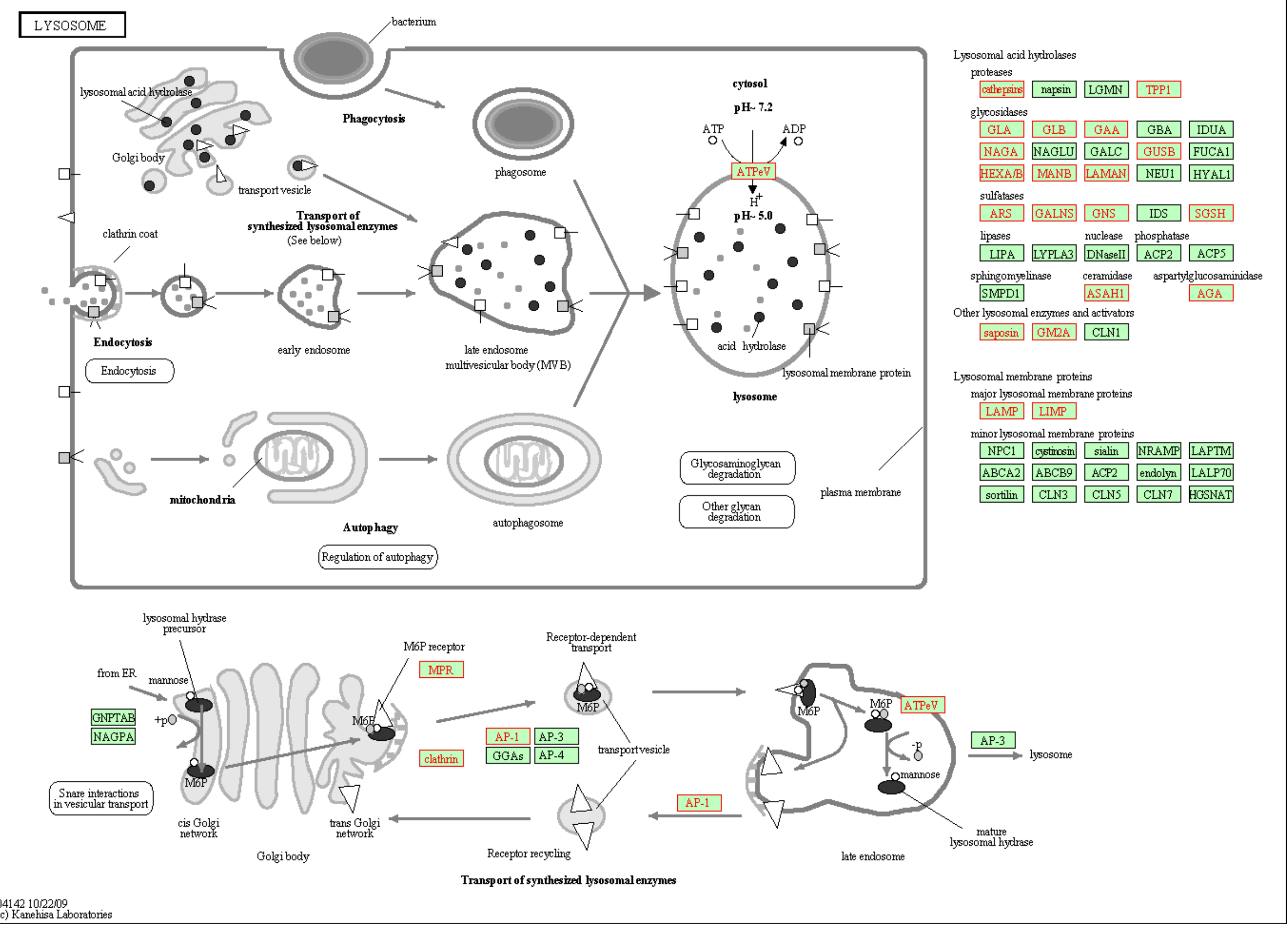




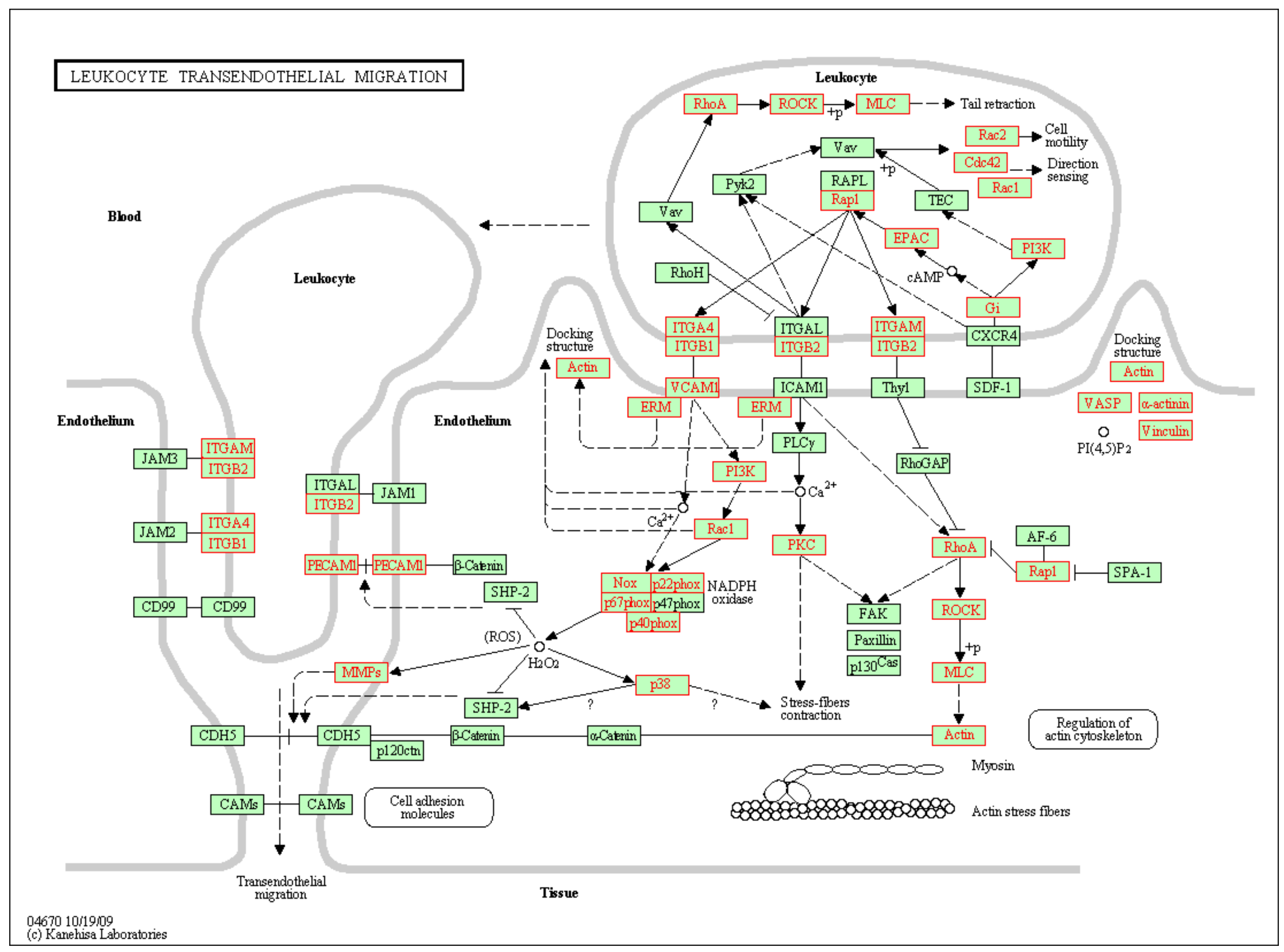




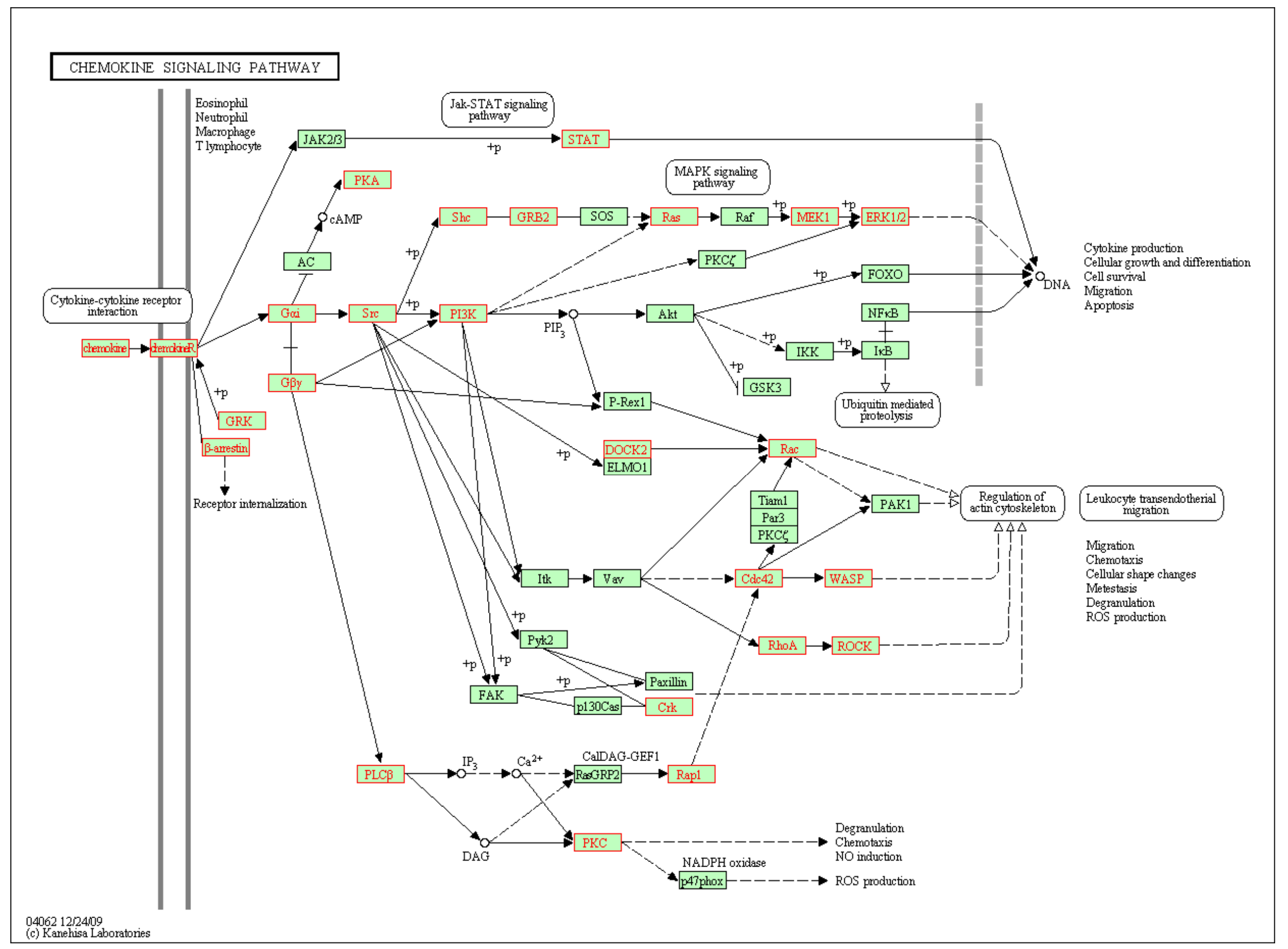




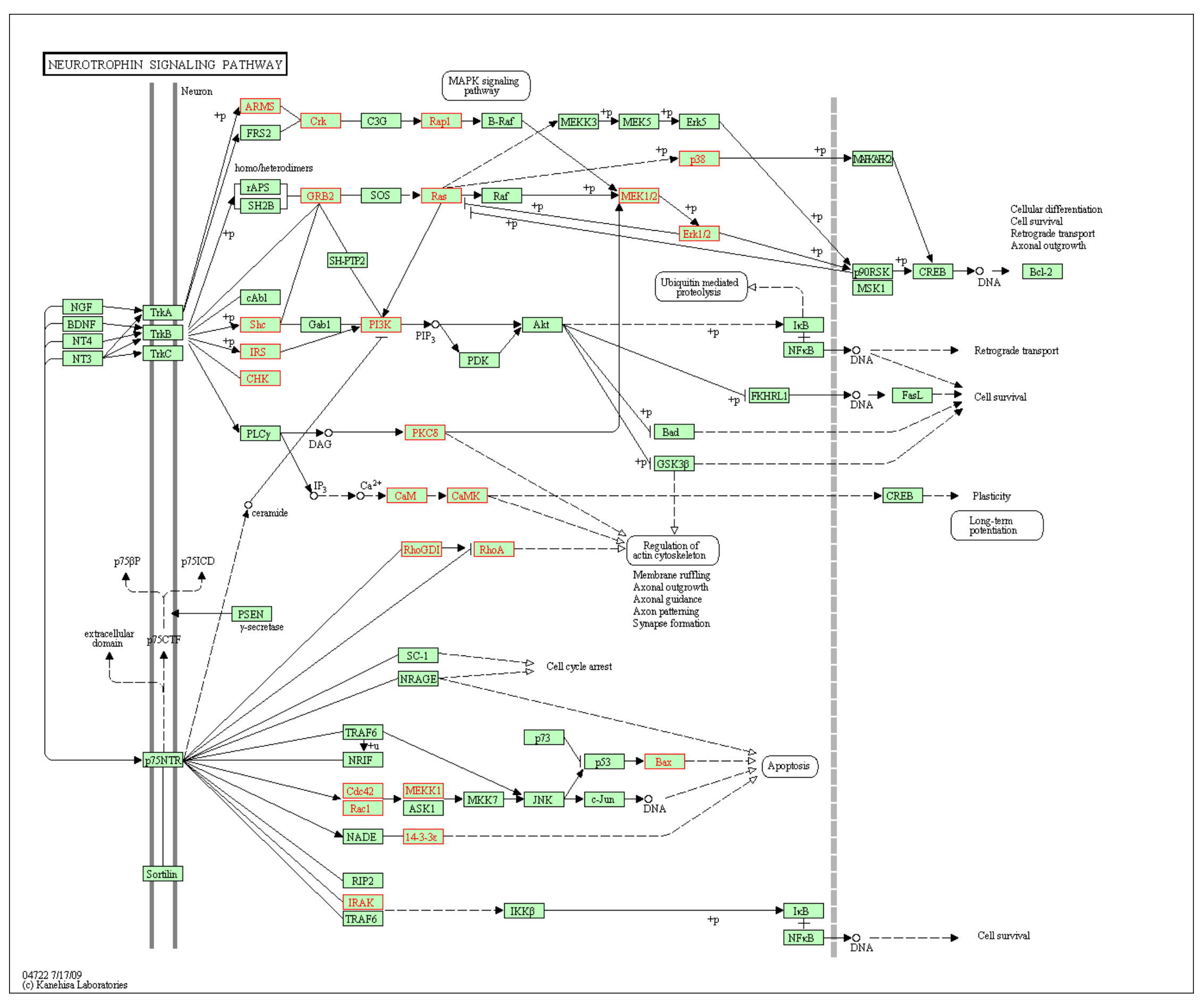


SYSTEMIC LUPUS ERYTHEMATOSUS

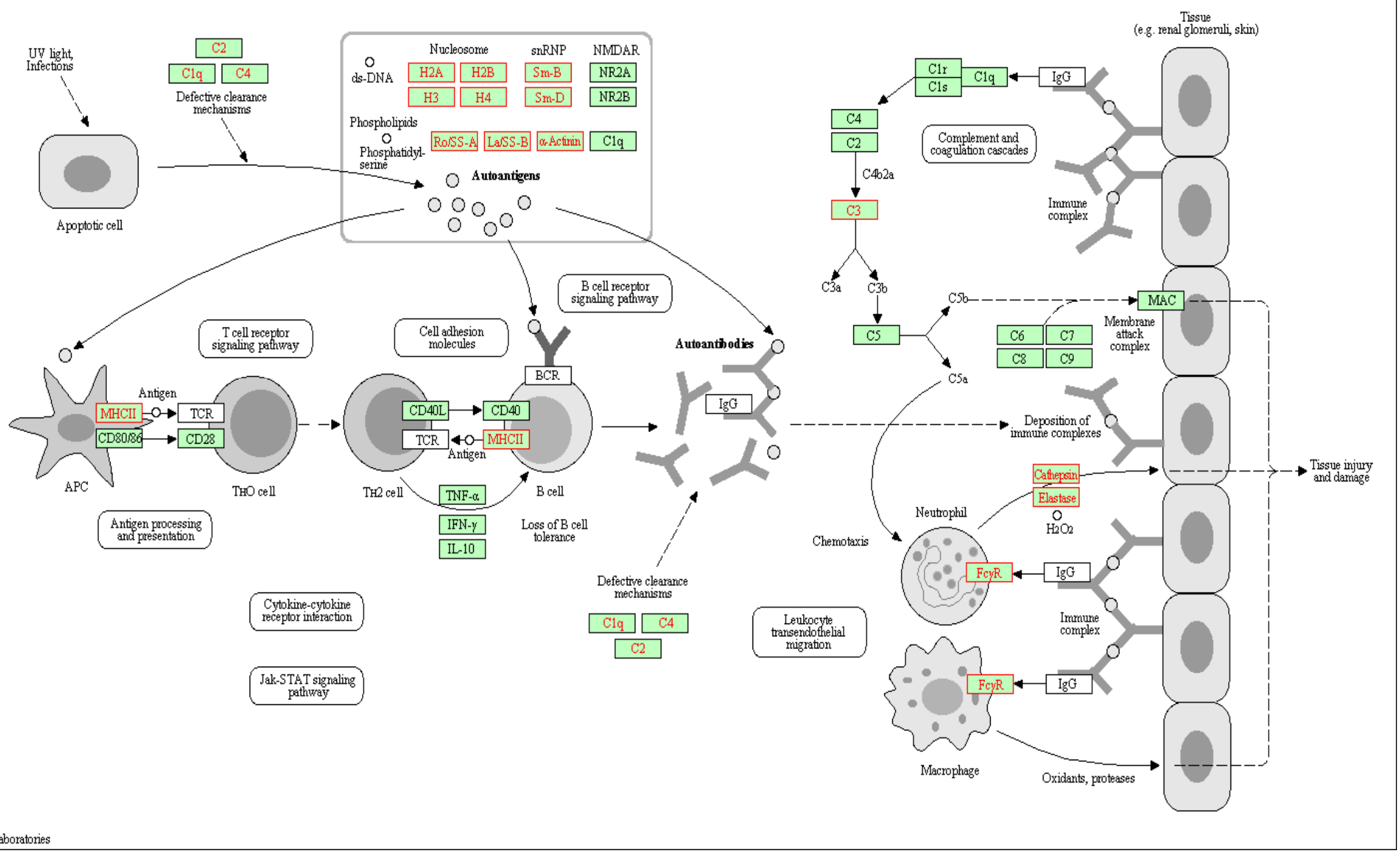


FCYR-MEDIATED PHAGOCYTOSIS

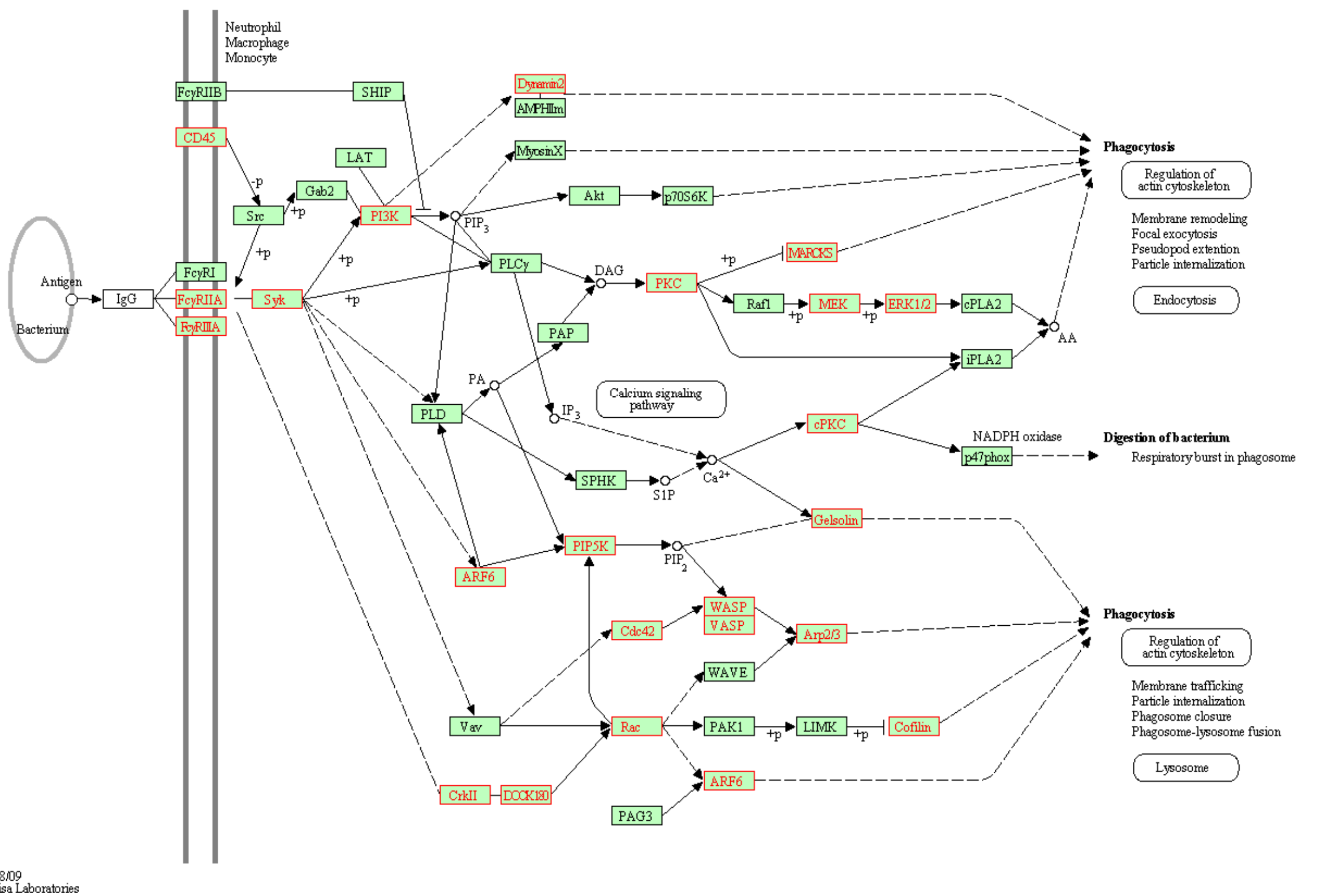




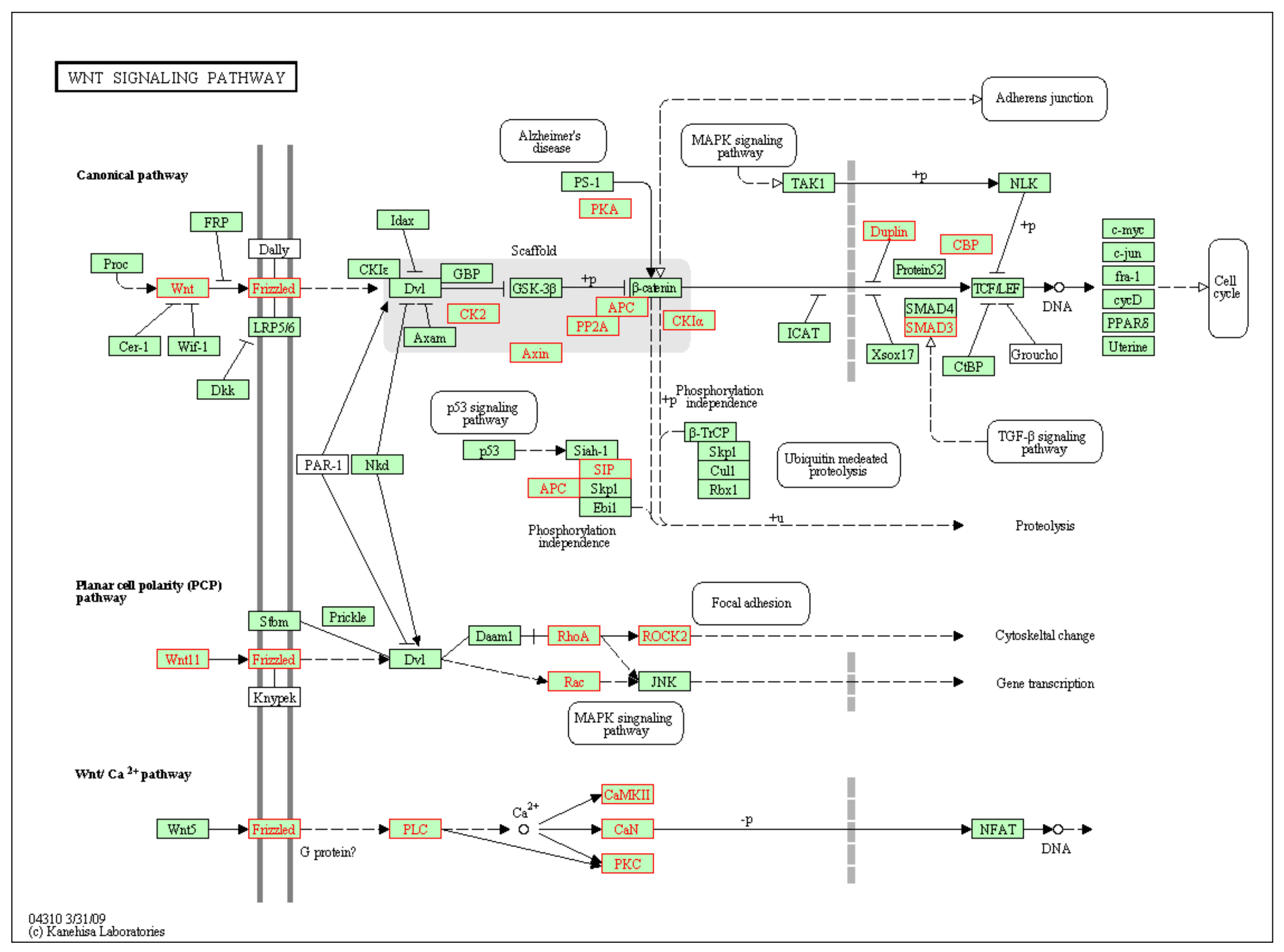




\section{PARKINSON'S DISEASE}

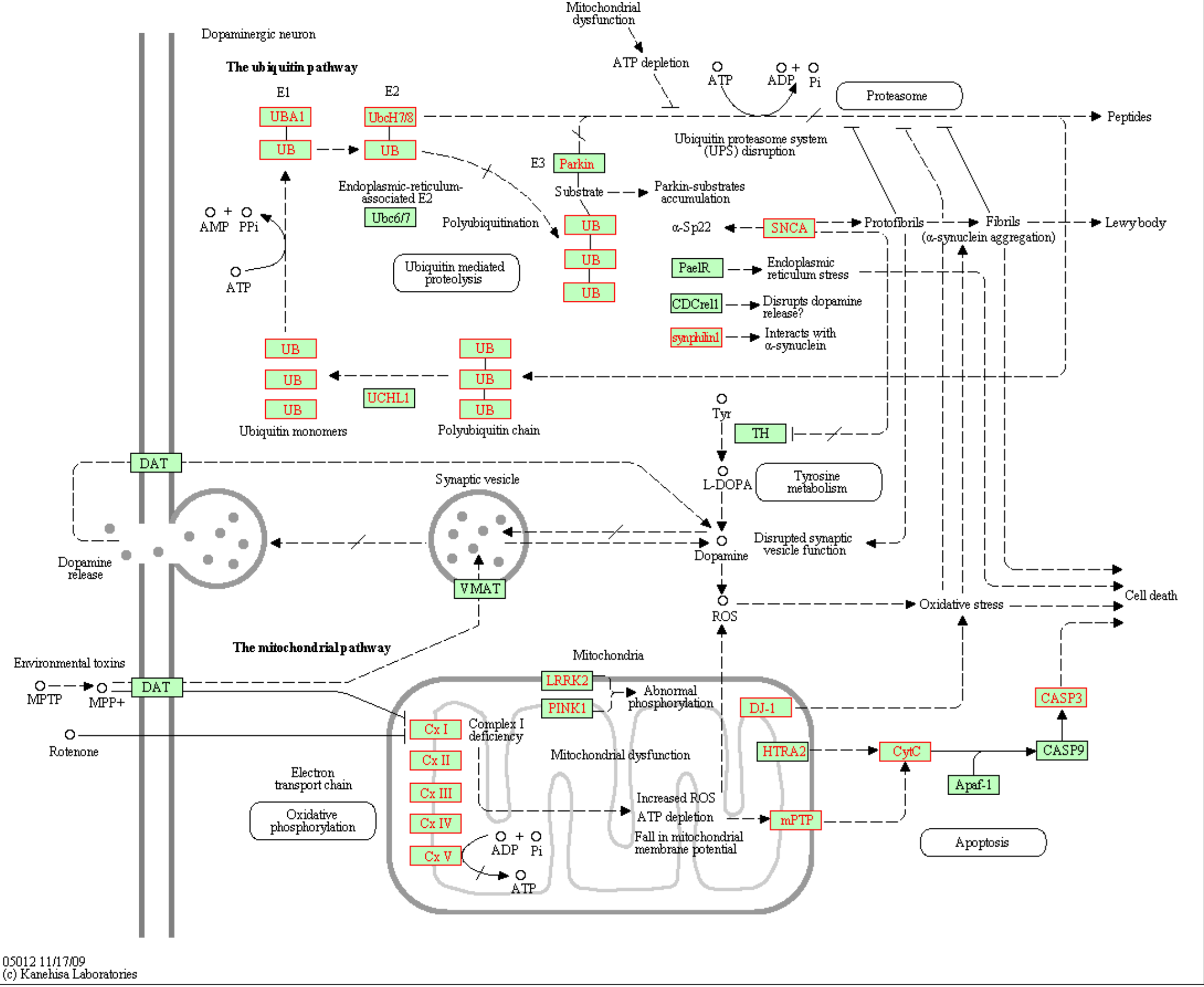


OXIDATIVE PHOSPHORYLATION

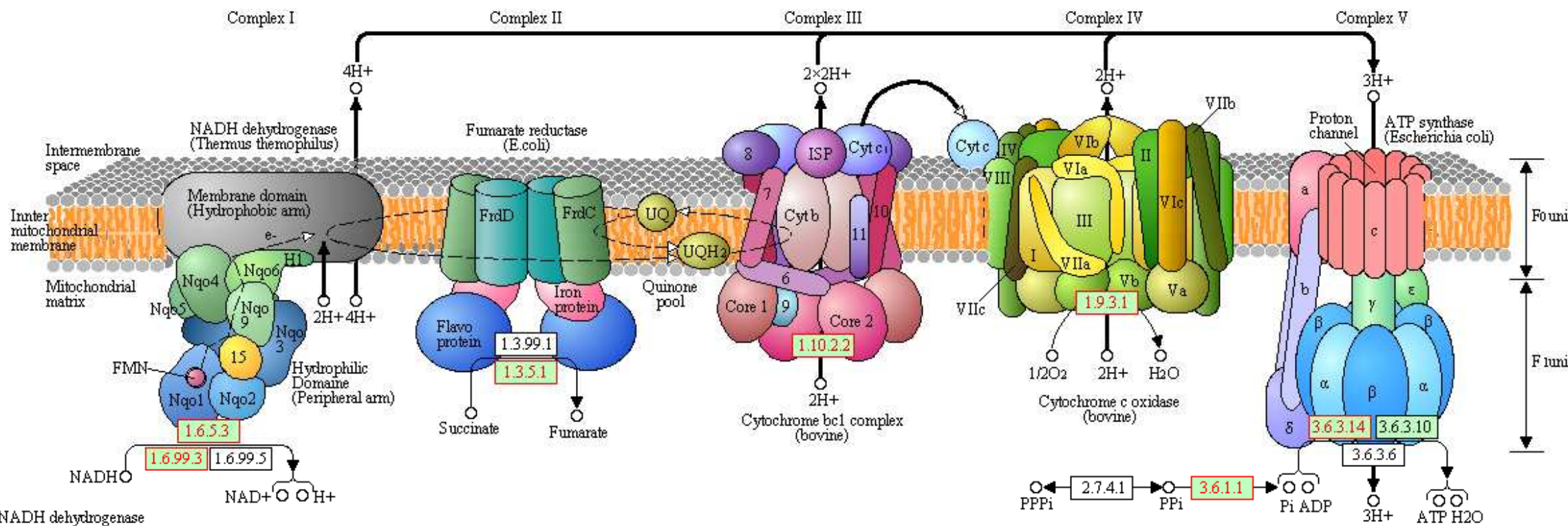

NADH dehydrogenase

$\mathrm{NAD}+\mathrm{OO} \mathrm{OH}$

\begin{tabular}{l|l|l|l|l|l|l|l|} 
E & ND1 & ND2 & ND3 & ND4 & ND4L & ND5 & ND6 \\
\hline
\end{tabular}

\begin{tabular}{|l|l|l|l|l|l|l|l|l|l|l|}
\hline Edufs1 & Ndufs2 & Ndufs3 & Ndufs4 & Ndufs5 & Ndufs6 & Ndufs? & Ndufs8 & Ndufv1 & Ndufv2 & Ndufv3 \\
\hline
\end{tabular}

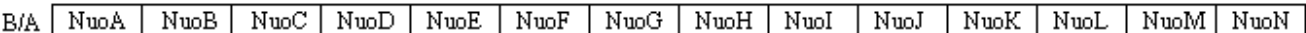

\begin{tabular}{l|l|l|l|l|l|l|l|l|l|l|l|l|l|l|l|l|l|} 
B/A & NdhC & NdhK & NdhJ & NdhH & NdhA & NdhL & NdhG & NdhE & NdhF & NdhD & NdhB & NdhL & NdhM & NdhL & HoxE & HoxF & HoxU \\
\hline
\end{tabular}

\begin{tabular}{|l|l|l|l|l|l|l|l|l|l|l|l|l|}
\hline E & Ndufa1 & Ndufa2 & Ndufa3 & Ndufa4 & Ndufas & Ndufa6 & Ndufa? & Ndufa8 & Ndufa9 & Ndufa10 & Ndufab1 & Ndufa11 \\
\hline
\end{tabular}

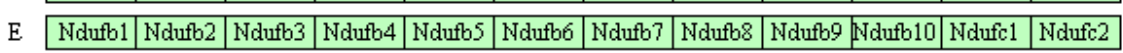

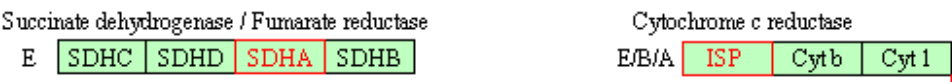

\begin{tabular}{|l|l|l|l|l|l|l|l|l|l|l|l|l|}
\hline BiA & SdhC & SdhD & SdhA & SdhB & E & COR1 & QCR2 & QCR6 & QCR7 & QCR8 & QCR9 & QCR10 \\
\hline
\end{tabular}

F-type ATPase (Bacteria)

\begin{tabular}{|l|l|l|l|l|l|l|l|}
\hline beta & alpha & garmma & delta & epsilon & c & a & b \\
\hline
\end{tabular}

F-type ATPase (Eukaryotes)

\begin{tabular}{|l|l|l|l|l|l|l|l|}
\hline beta & alpha & gamura & OSCP & delta & epsilon & c & a \\
\hline
\end{tabular} \begin{tabular}{|c|c|c|c|c|c|}
\hline b & e & fo & f & 8 & \\
\hline d & f & h & j & k & g \\
\hline
\end{tabular}

F-type ATPase (Prokaryotes)

\begin{tabular}{|l|l|l|l|l|l|l|l|}
\hline A & B & C & D & E & F & I & K \\
\hline
\end{tabular}

F-type ATPase (Eukaryotes)

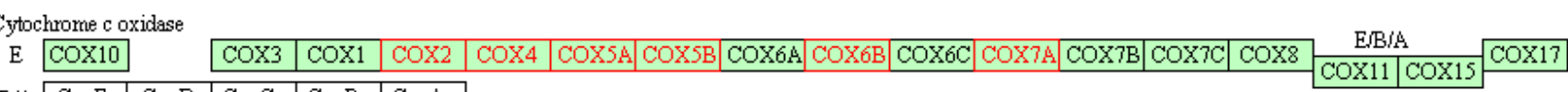

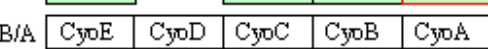

B/A \begin{tabular}{|l|l|l|l|l|} 
CyoE & CyoD & CyoC & CyoB & CyoA \\
\hline & CoxD & CoxC & CoxA & CoxB \\
\hline
\end{tabular} \begin{tabular}{|l|l|l|l|} 
CoxD & CoxC & CoxA & CoxB \\
\hline QoxD & QoxC & QoxB & QoxA \\
\hline
\end{tabular}

Cytochrome c oxidase, cbb3-type

Cytochrome bd complex

\begin{tabular}{|l|l|}
\hline B/A CydA & CydB \\
\hline
\end{tabular}

\begin{tabular}{|c|c|c|c|c|c|c|c|}
\hline A & B & C & D & E & F & G & H \\
\hline I & AC 39 & $54 k \mathrm{D}$ & S1 & lipid & & & \\
\hline
\end{tabular}




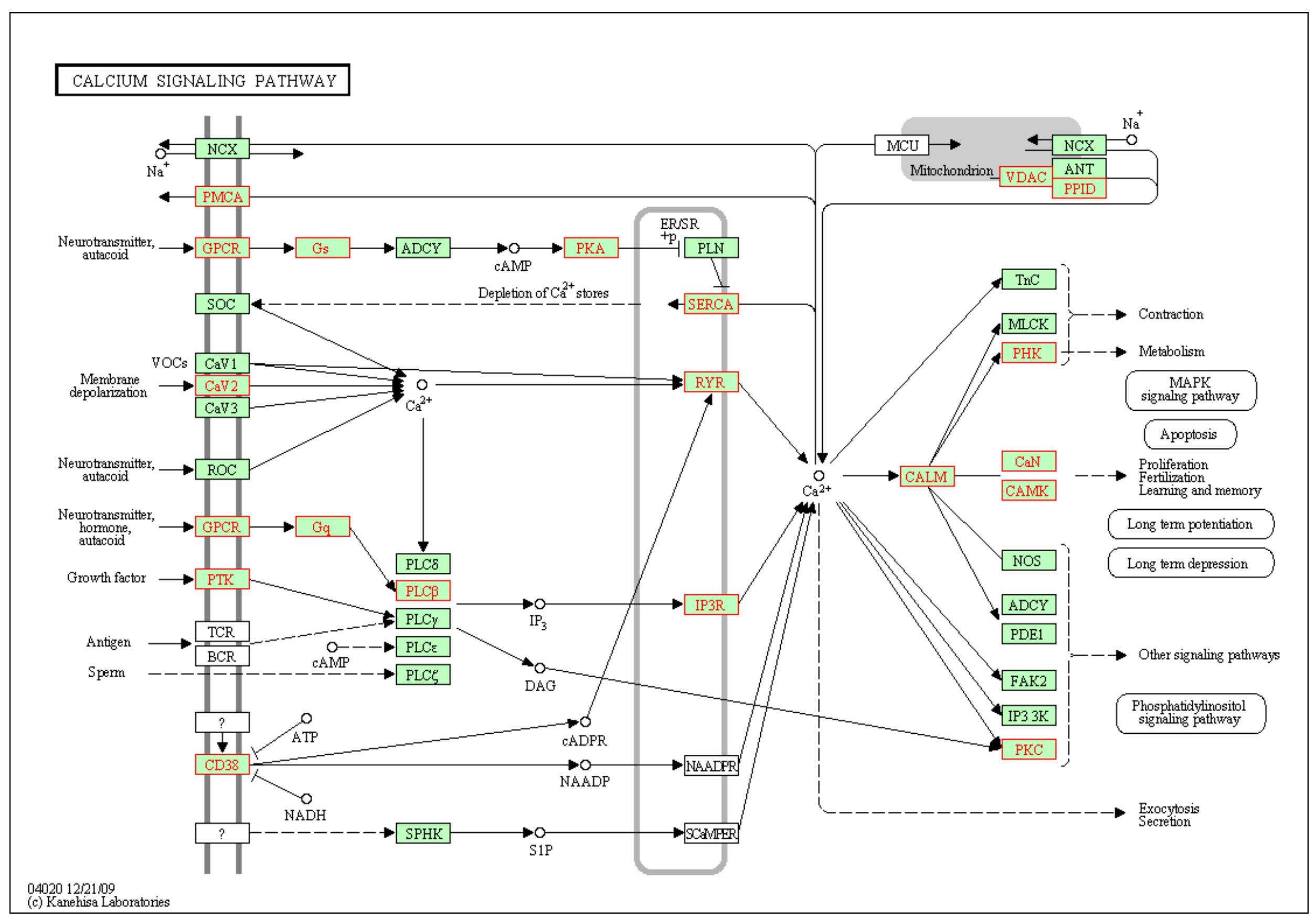


RIBOSOME

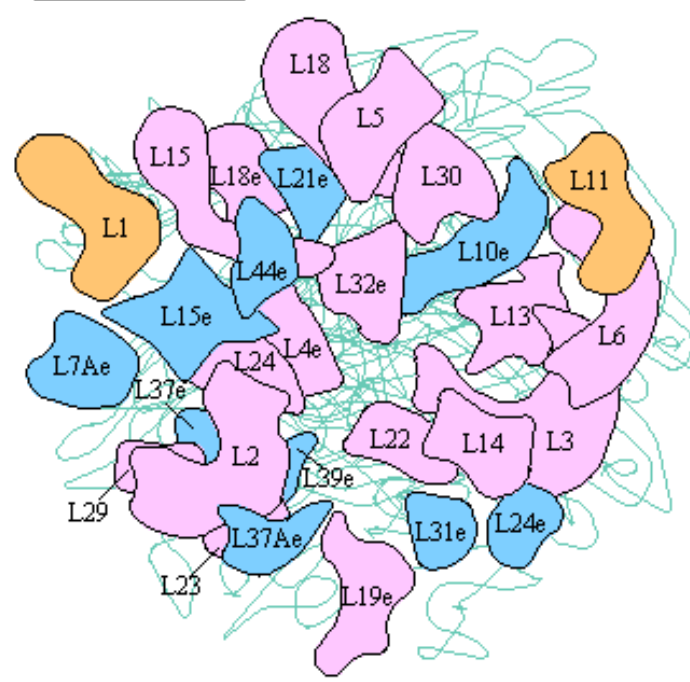

Large suburit (Haloarcula marismortui)

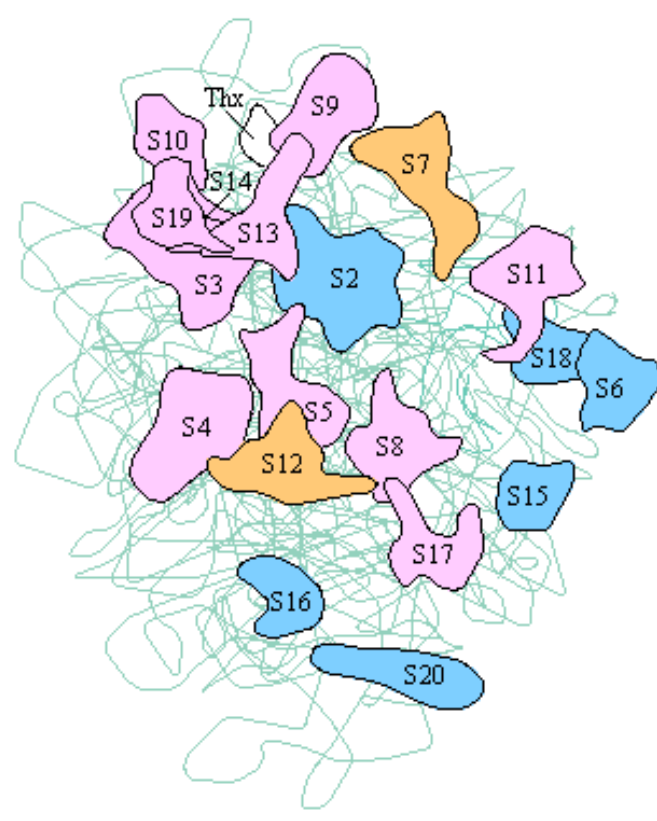

Small suburit (Thermus aquaticus)
Ribosomal RNAs

\section{Bacteria / Archae:}

Eukaryotes

\begin{tabular}{|l|l|l|}
\hline $23 \mathrm{~S}$ & $5 \mathrm{~S}$ & \\
\hline $25 \mathrm{~S}$ & $5 \mathrm{~S}$ & $5.8 \mathrm{~S}$ \\
\hline
\end{tabular} \begin{tabular}{|l|}
\hline $16 \mathrm{~S}$ \\
\hline $18 \mathrm{~S}$ \\
\hline
\end{tabular}

\section{Ribosomal proteins}

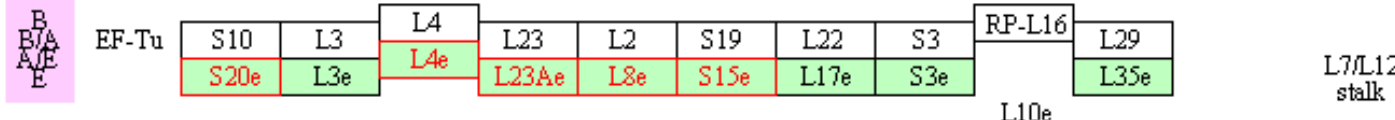

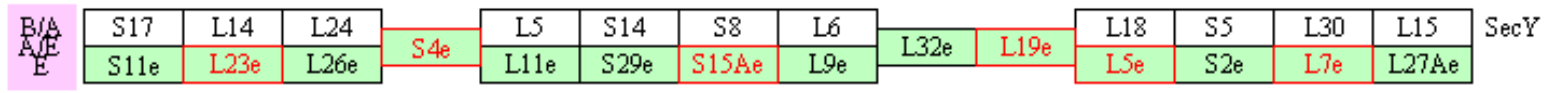

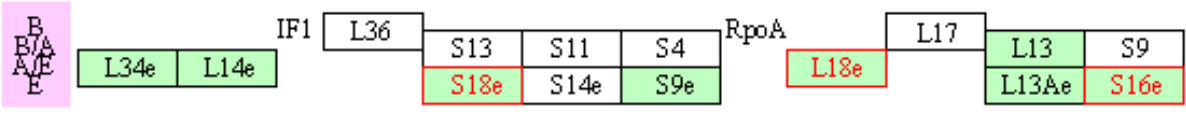

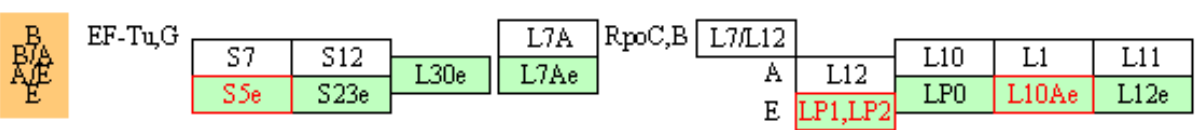

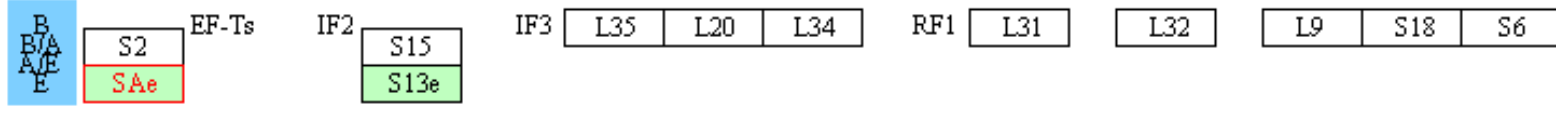

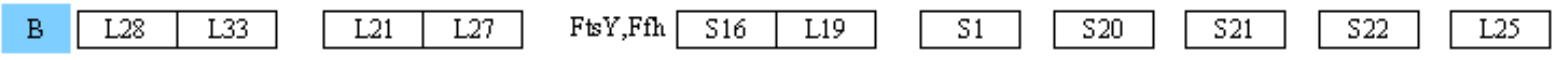
A.F \begin{tabular}{|l|l|l|l|l|l|l|l|l|l|l|l|l|}
\hline L10e & L13e & L15e & L21e & L24e & L31e & L3SAe & L37e & L37Ae & L39e & L40e & LA1e & L44e \\
\hline
\end{tabular}

A/E \begin{tabular}{|l|l|l|l|l|l|l|l|l|l|l|l|l|}
\hline S3Ae & S6e & S8e & S17e & S19e & S24e & S25e & S26e & S27e & S27Ae & S28e & S30e & A \\
\hline
\end{tabular}

E L L6e \begin{tabular}{|l|l|l|l|l|l|l|}
\hline L18Ae & L22e & L27e & L28e & L29e & L36e & L38e \\
\hline
\end{tabular}

\begin{tabular}{l|l|l|l|l|}
\hline E & S7e & S10e & S12e & S21e \\
\cline { 2 - 4 }
\end{tabular} 


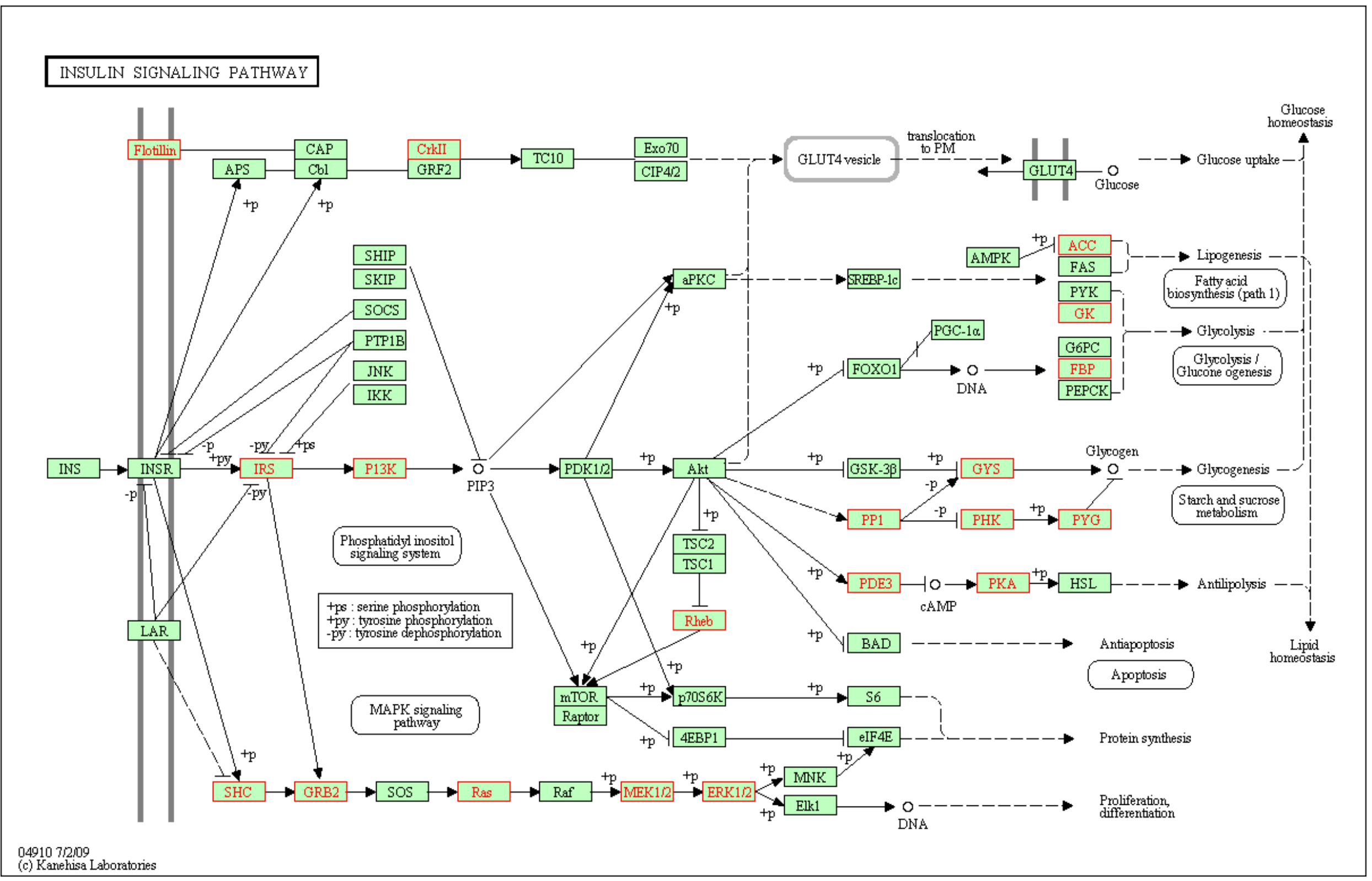




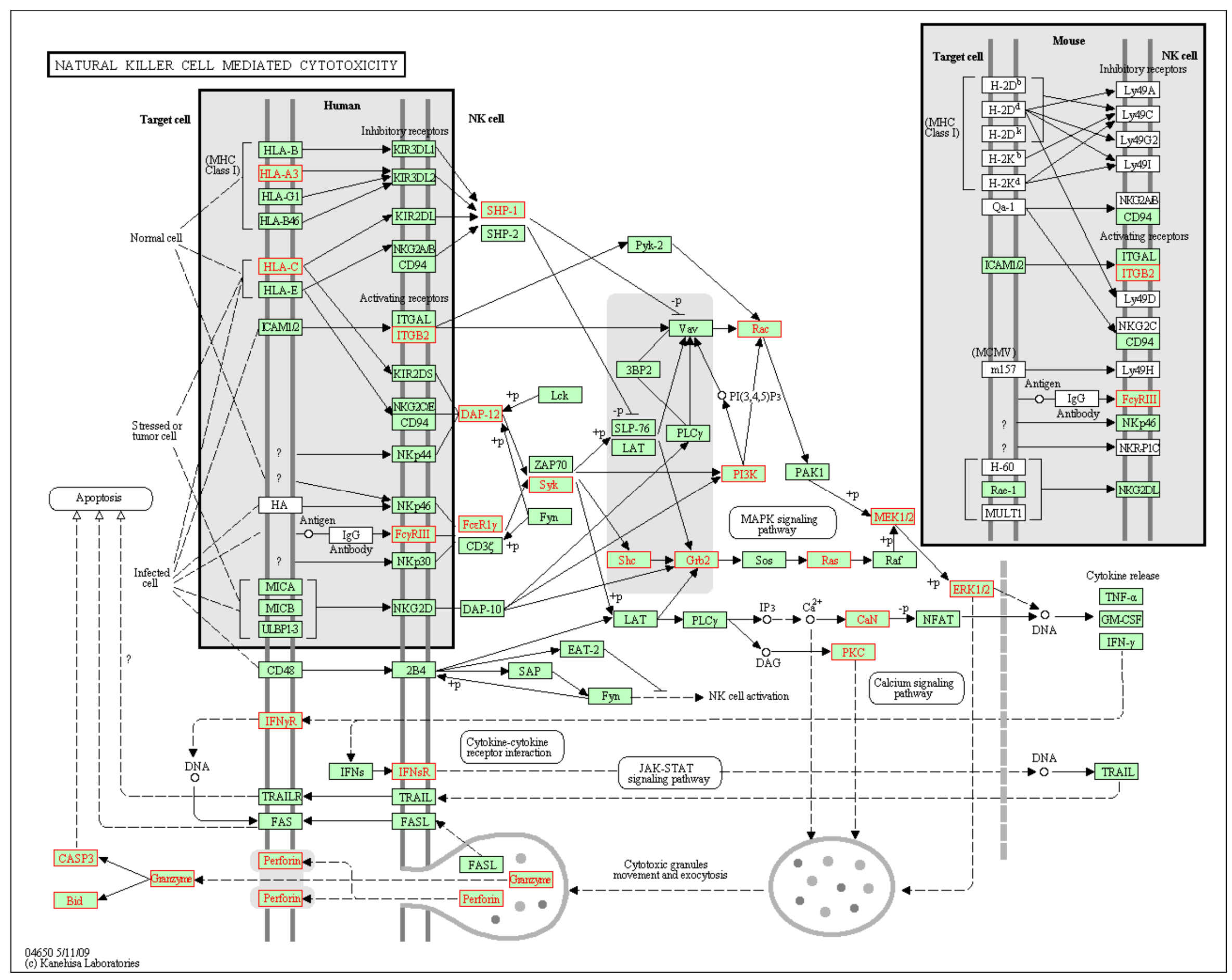




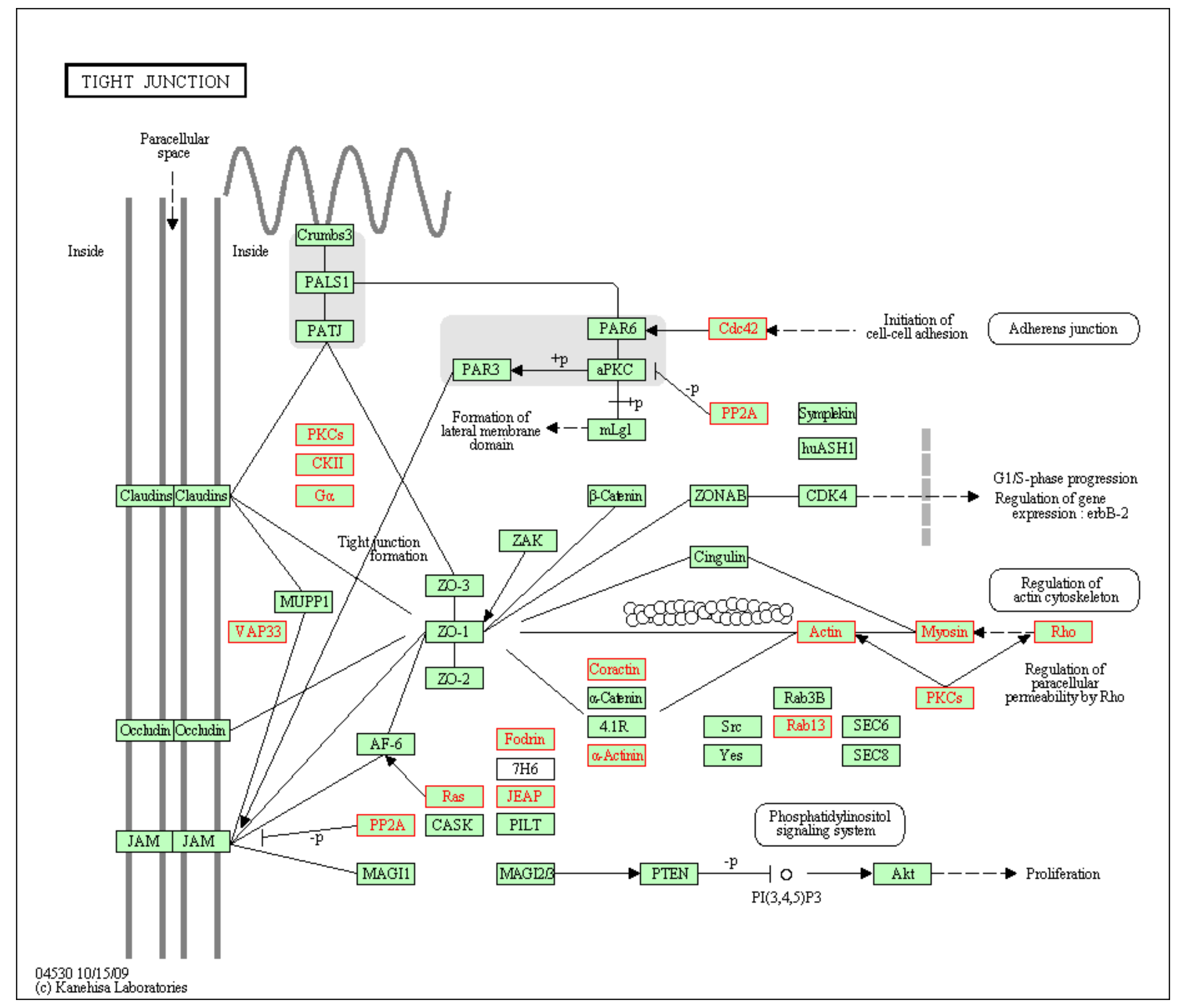




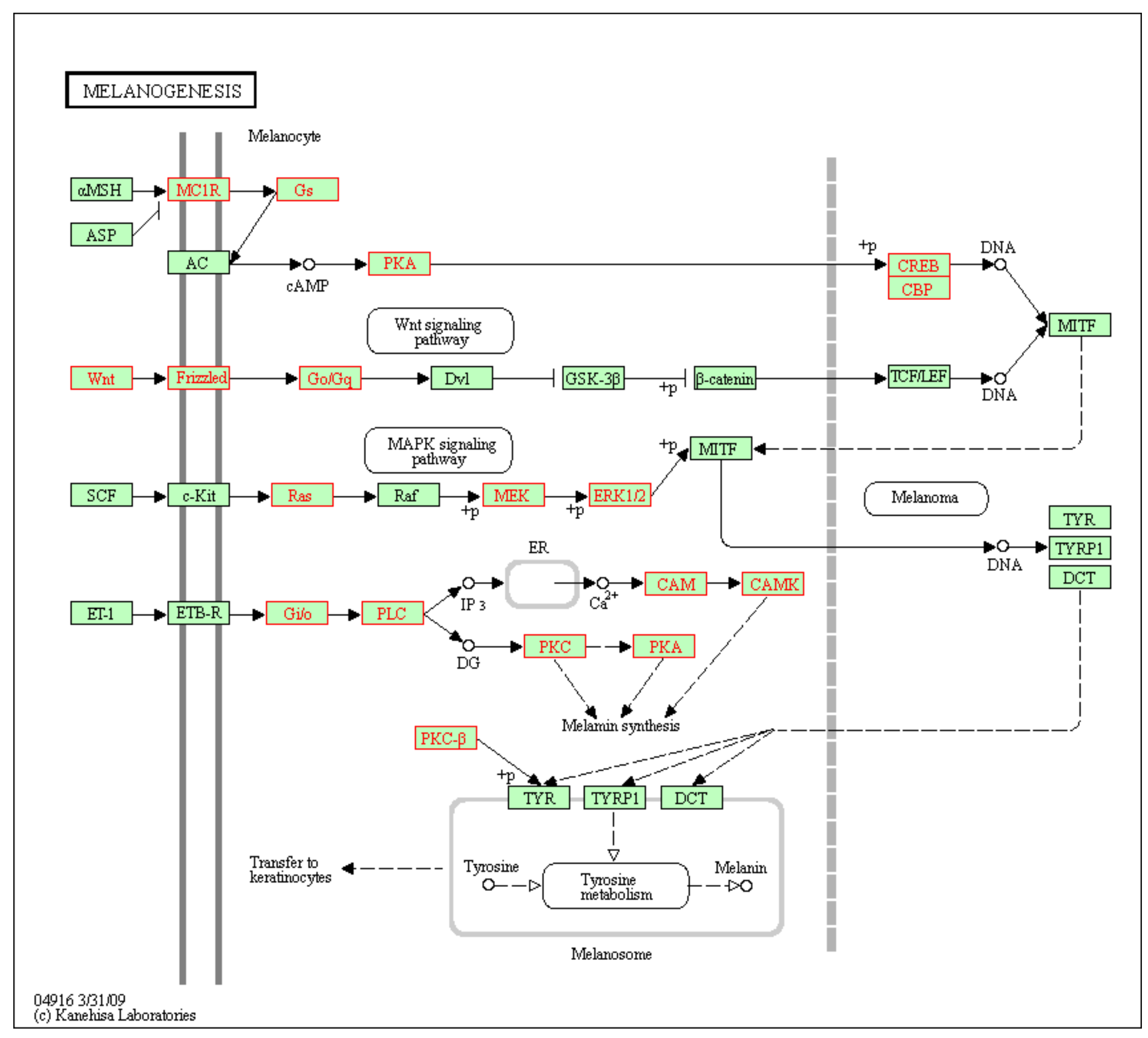




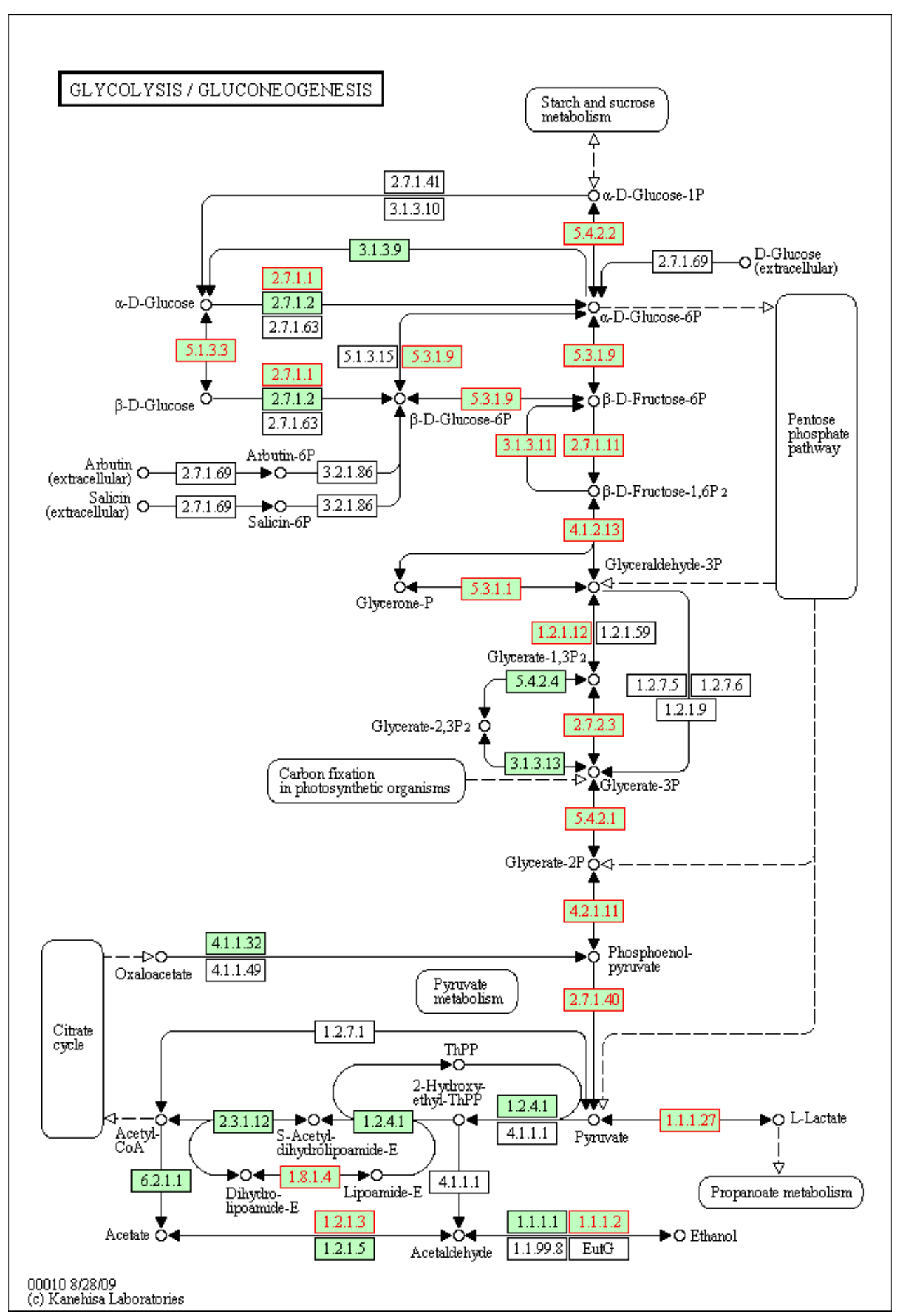




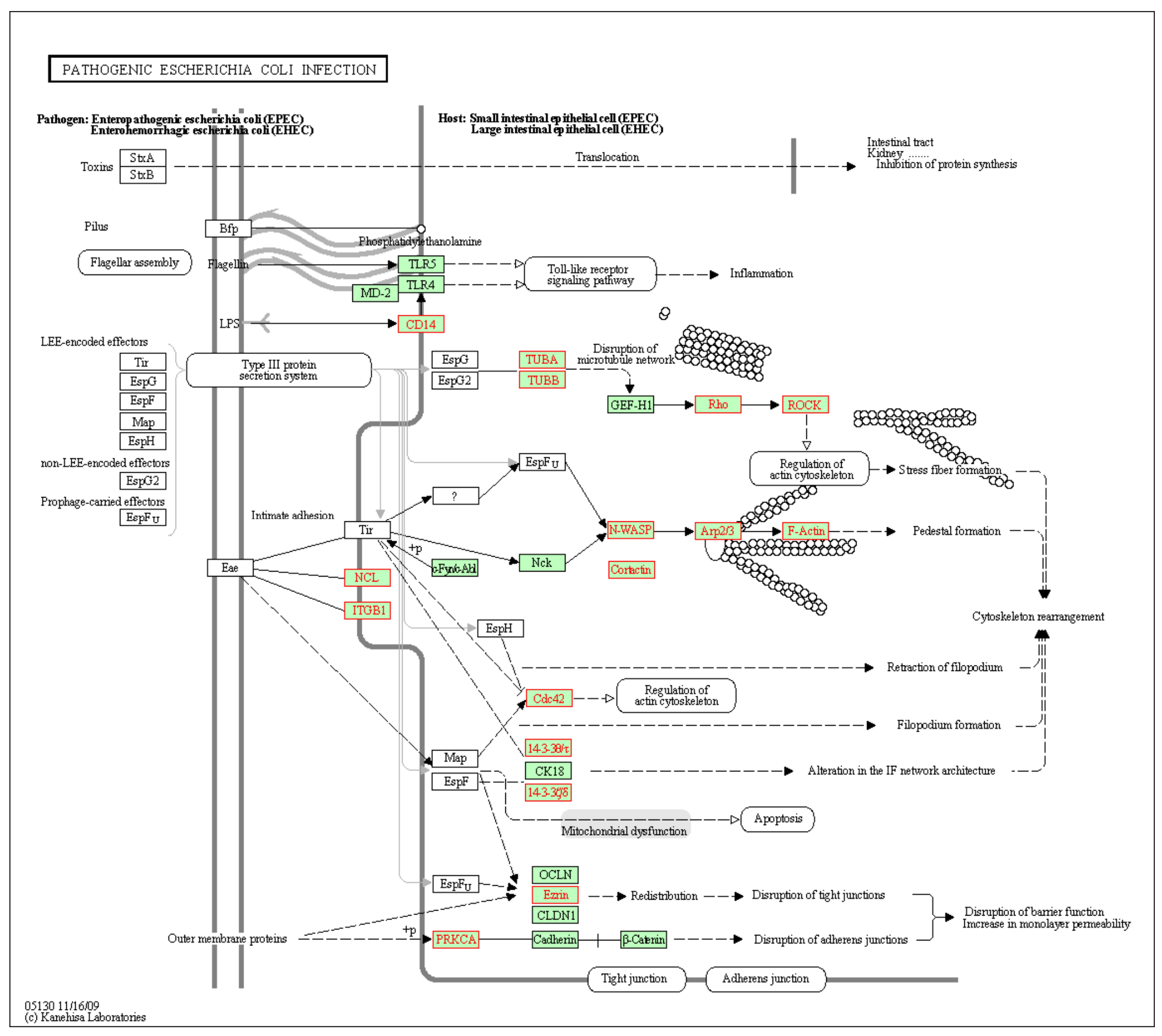




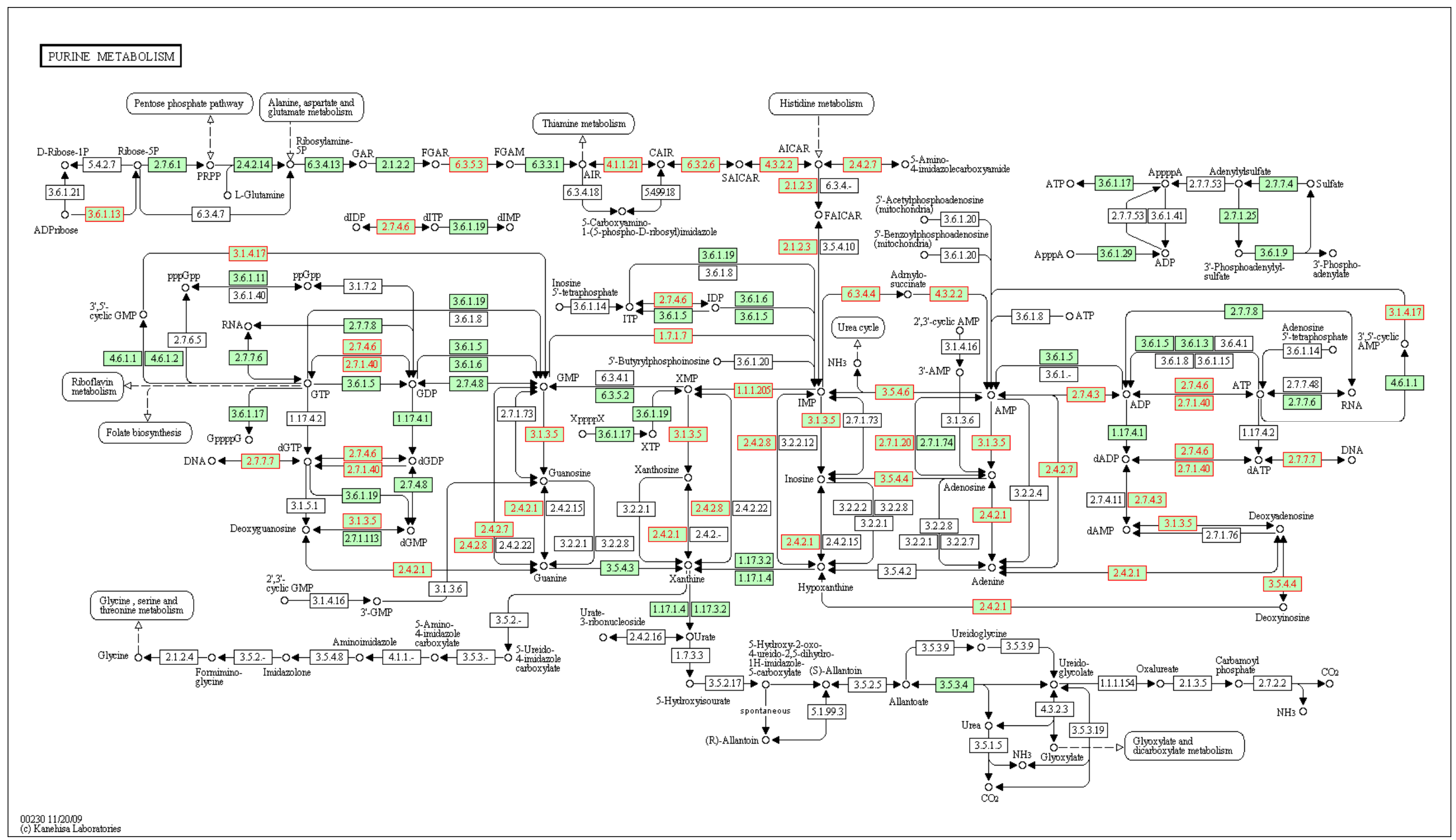




\section{LONG-TERM POTENTIATION}

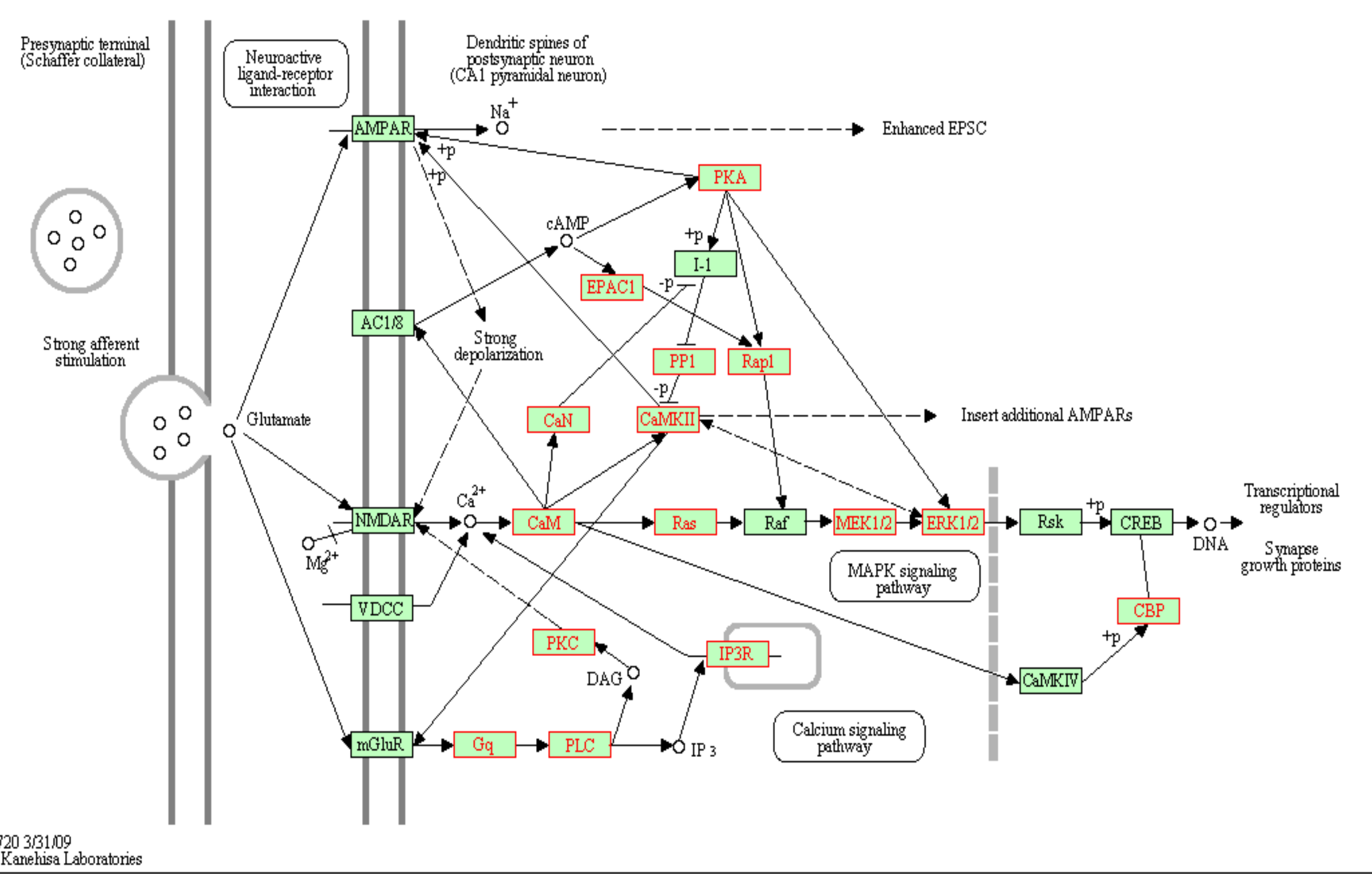




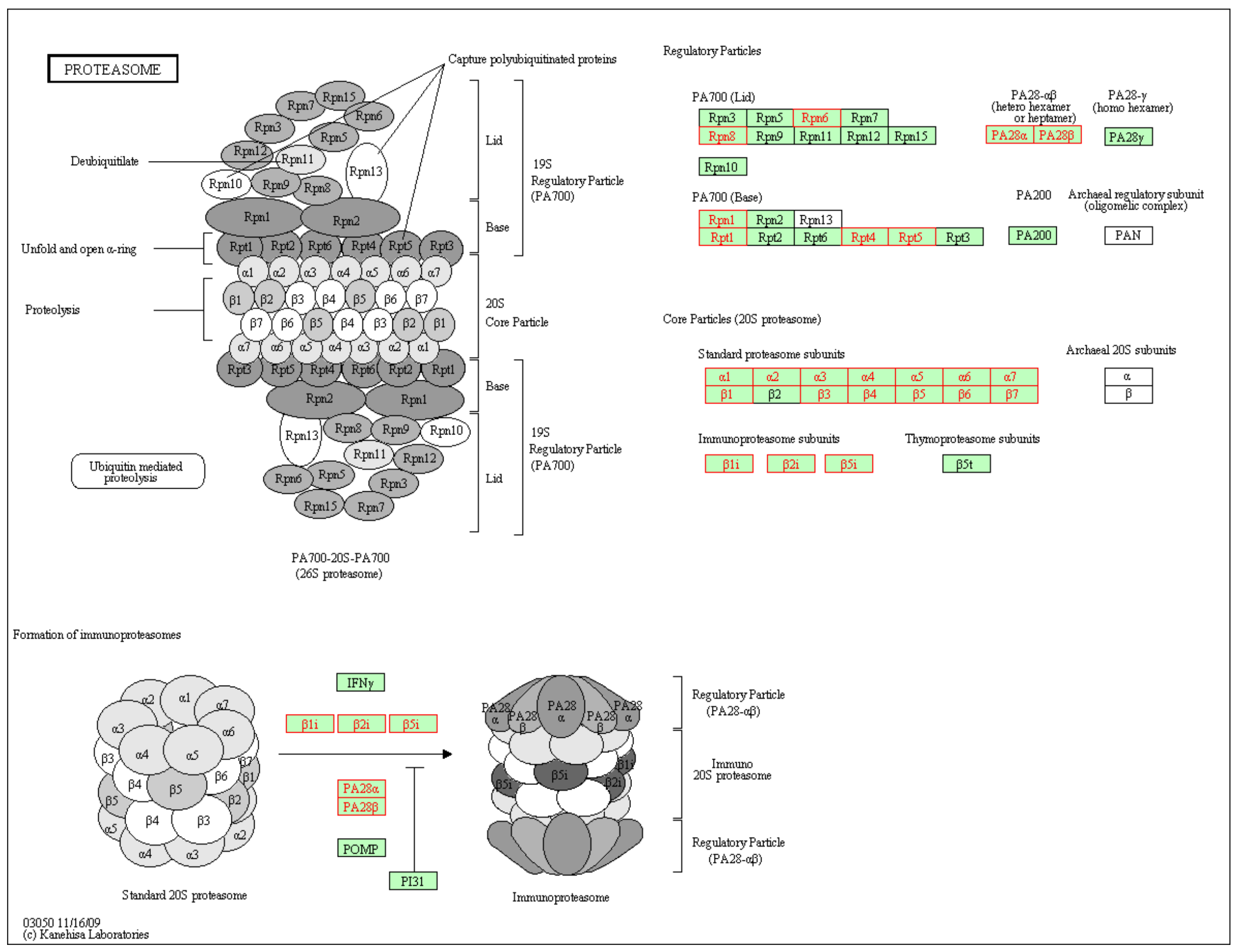




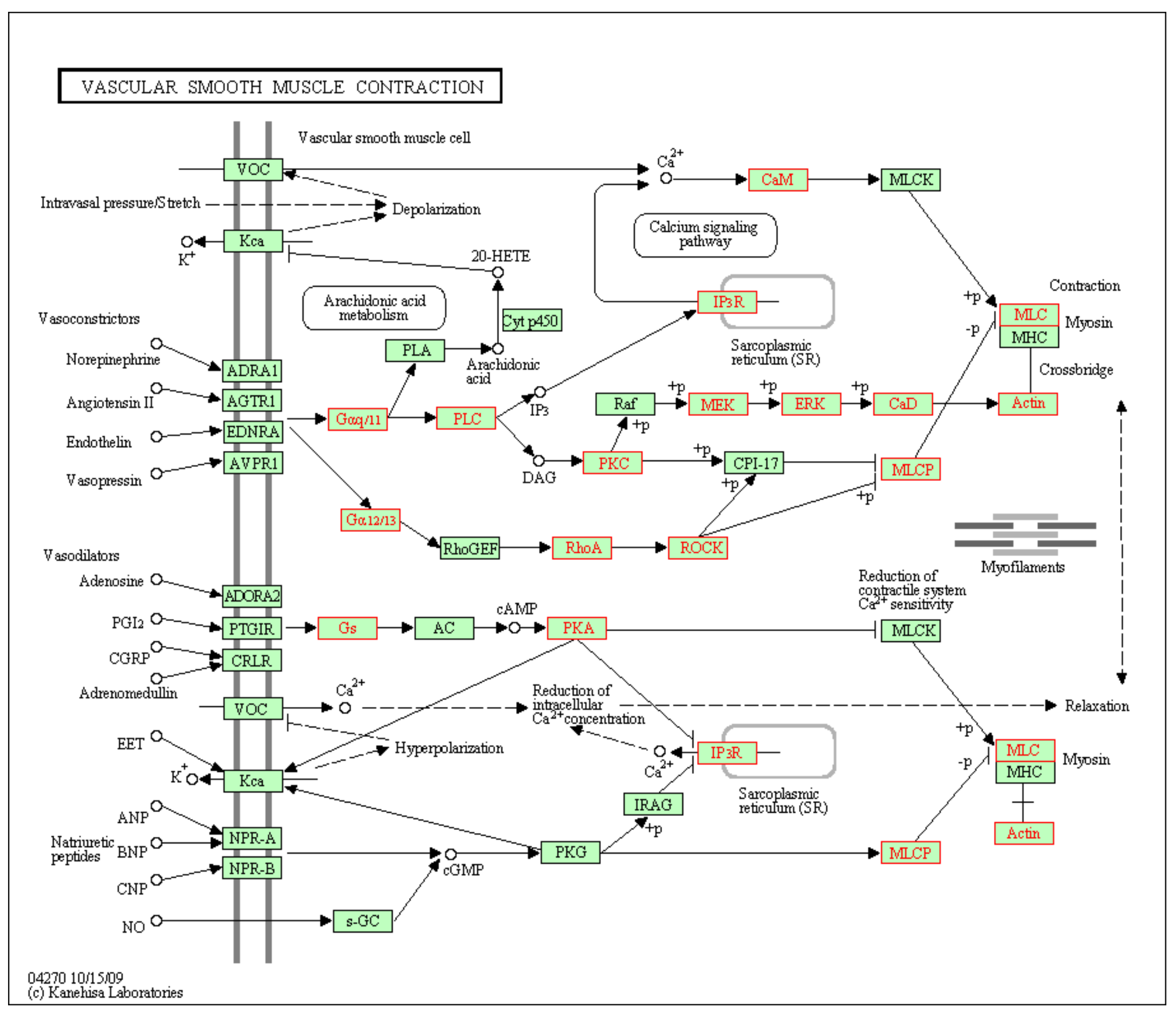




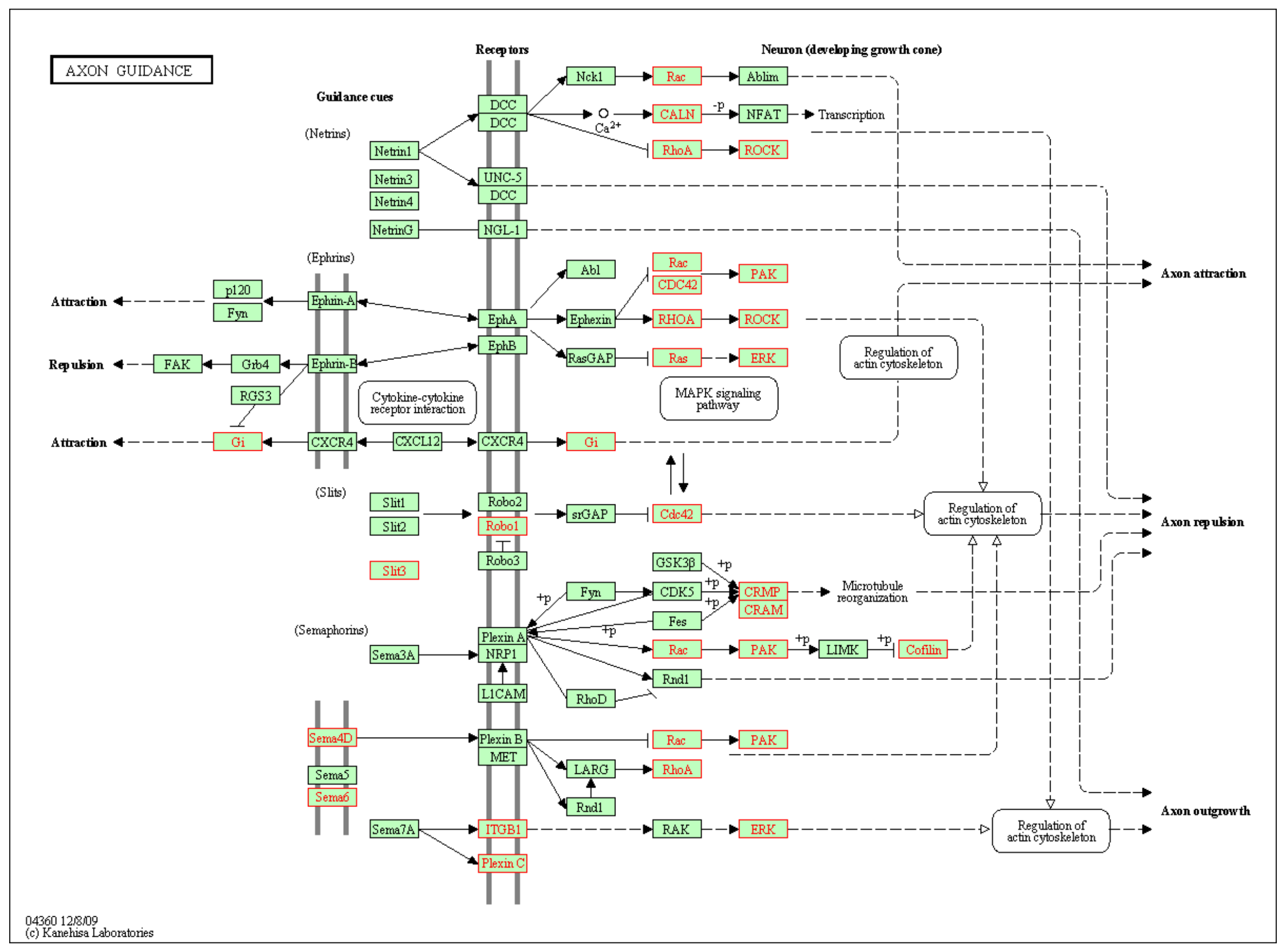




\section{OOCYTE MEIOSIS}

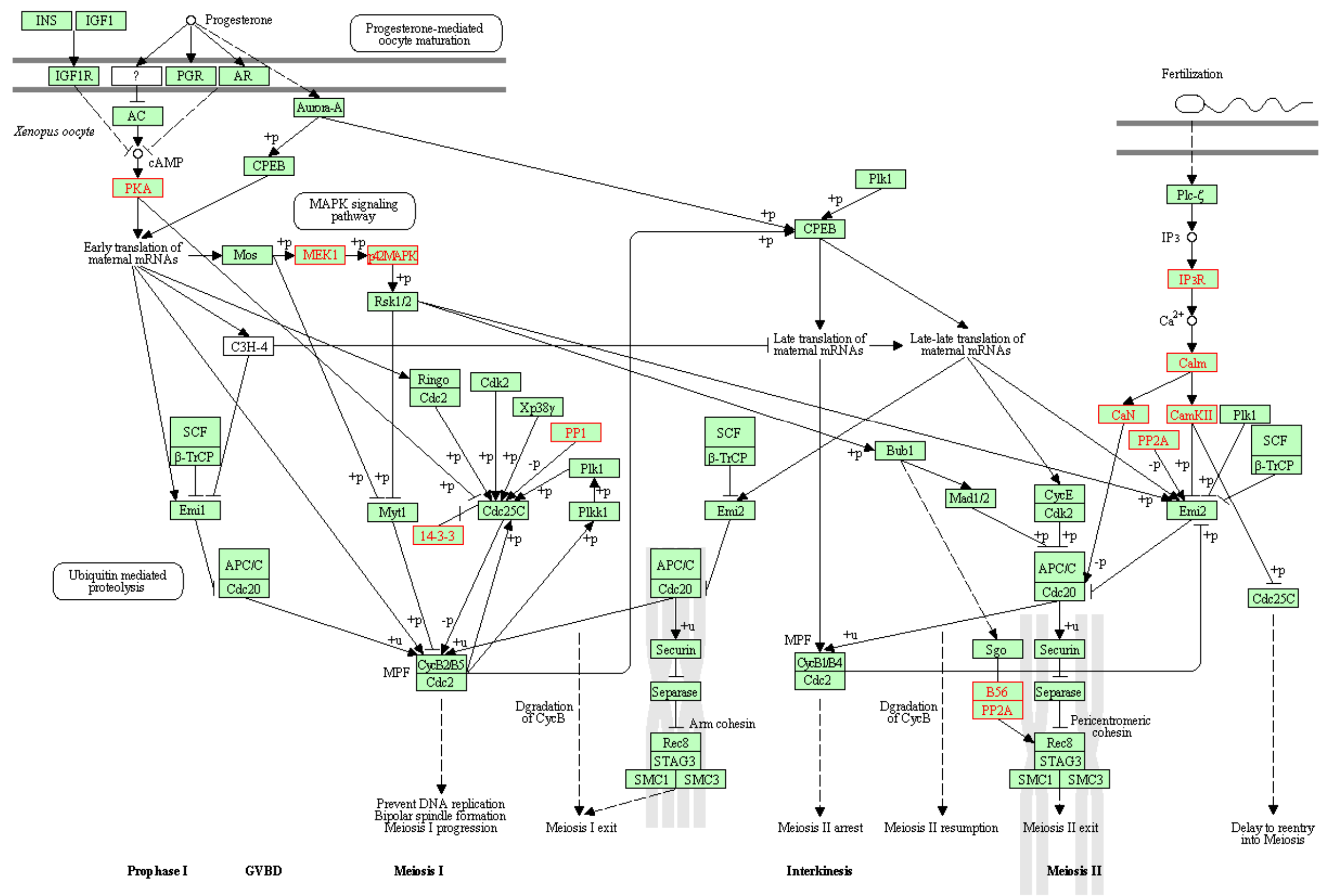




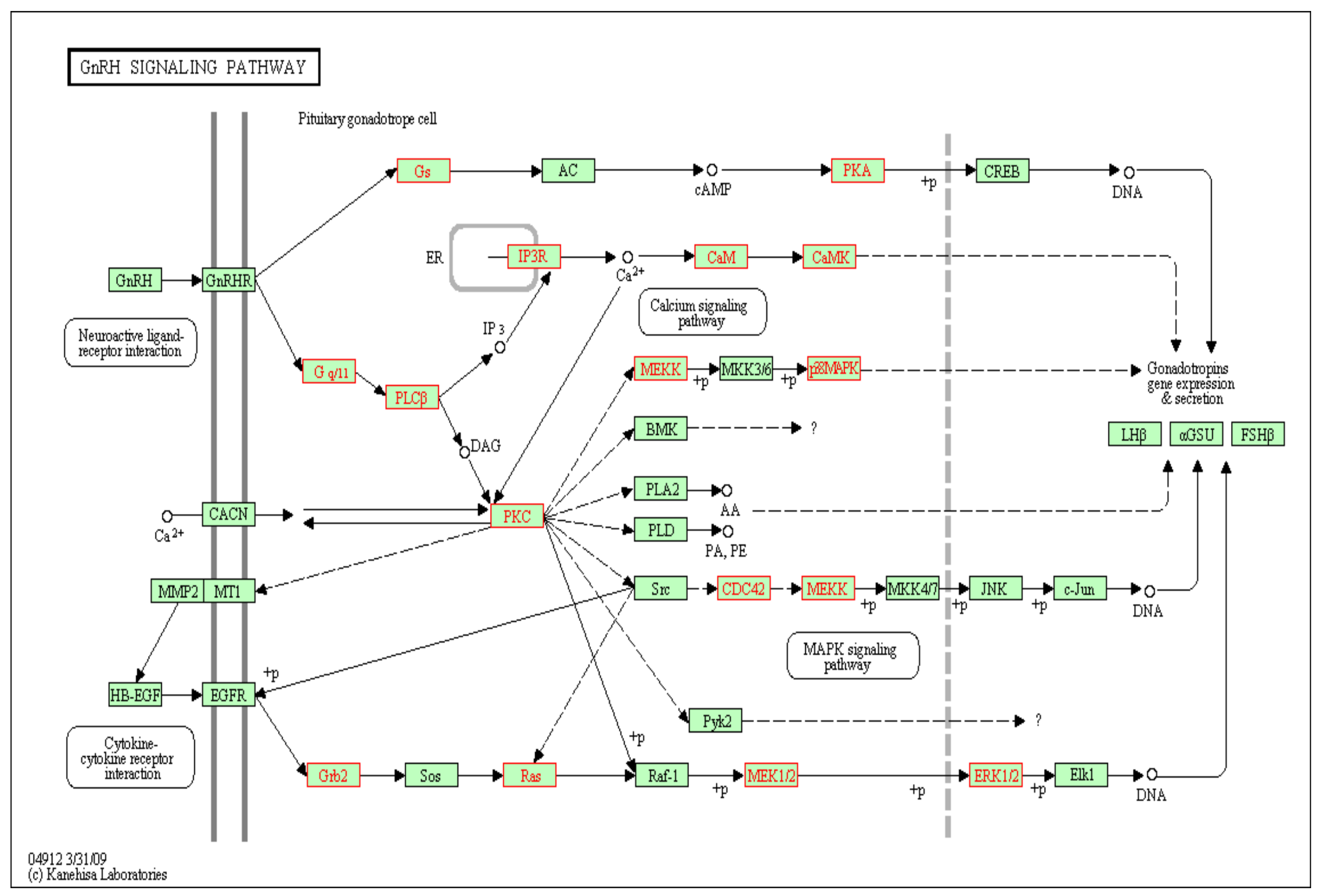




\section{GAP JUNCTION}
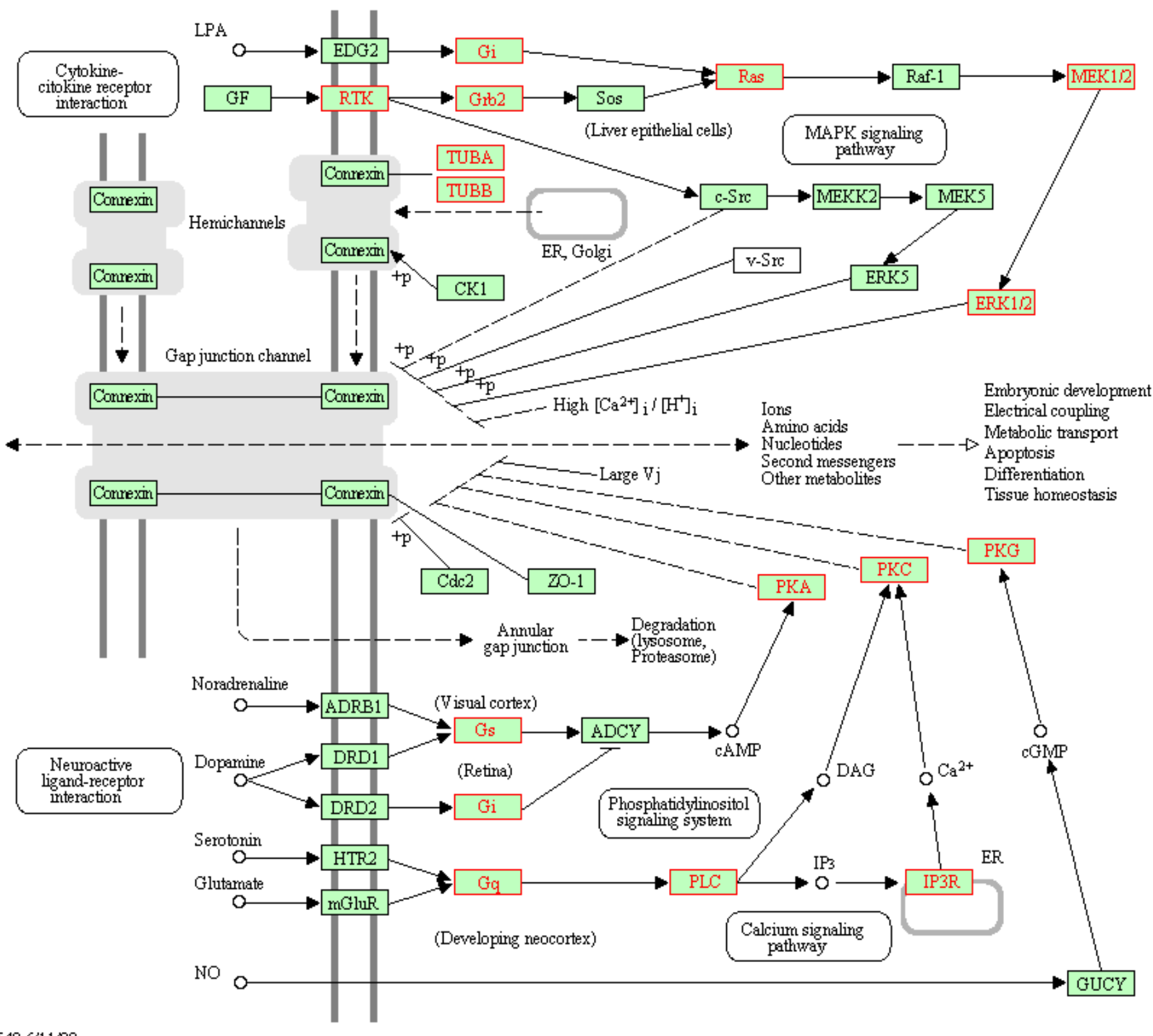
ECM-RECEPTOR INTERACTION

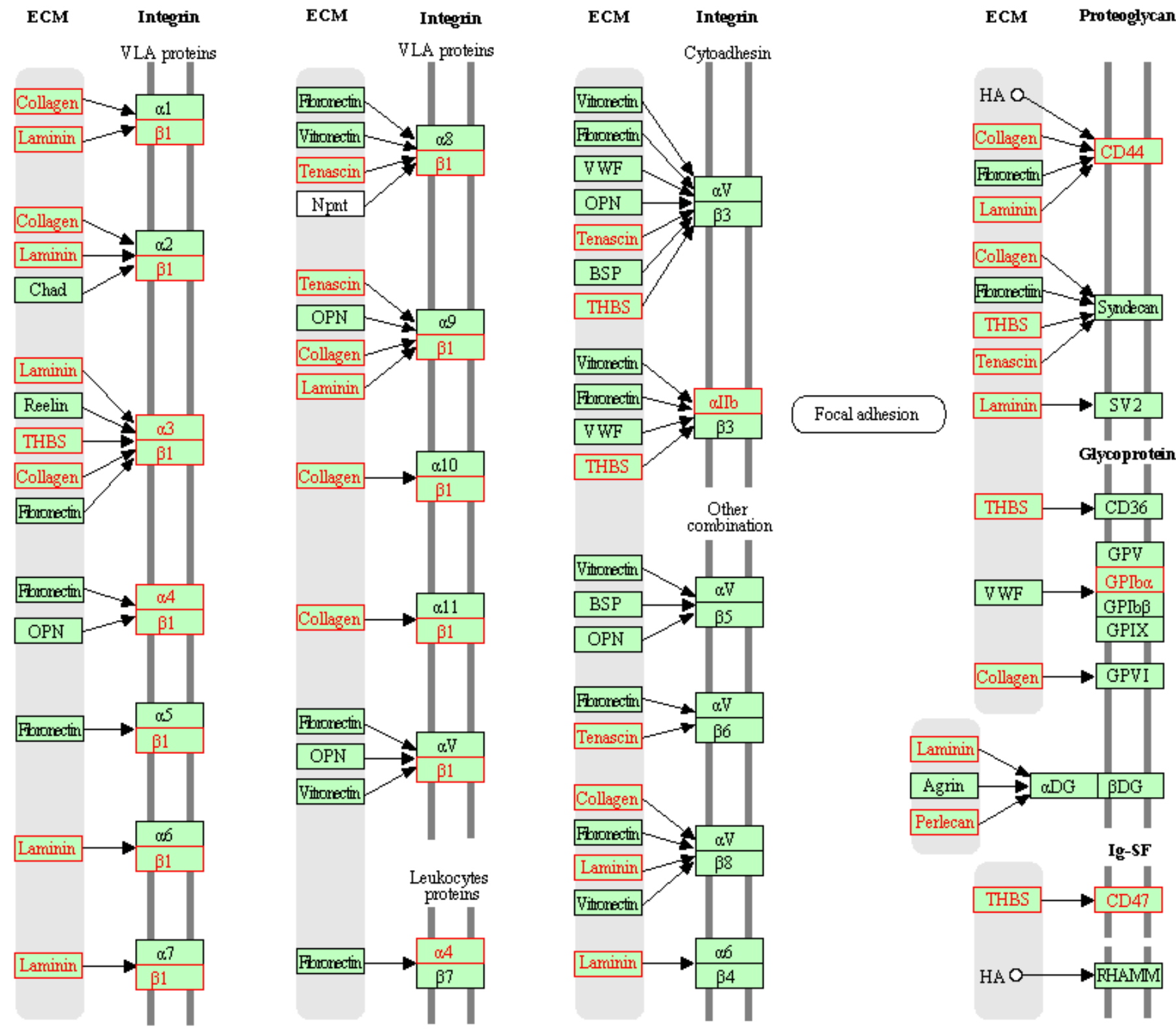


ADHERENS JUNCTION

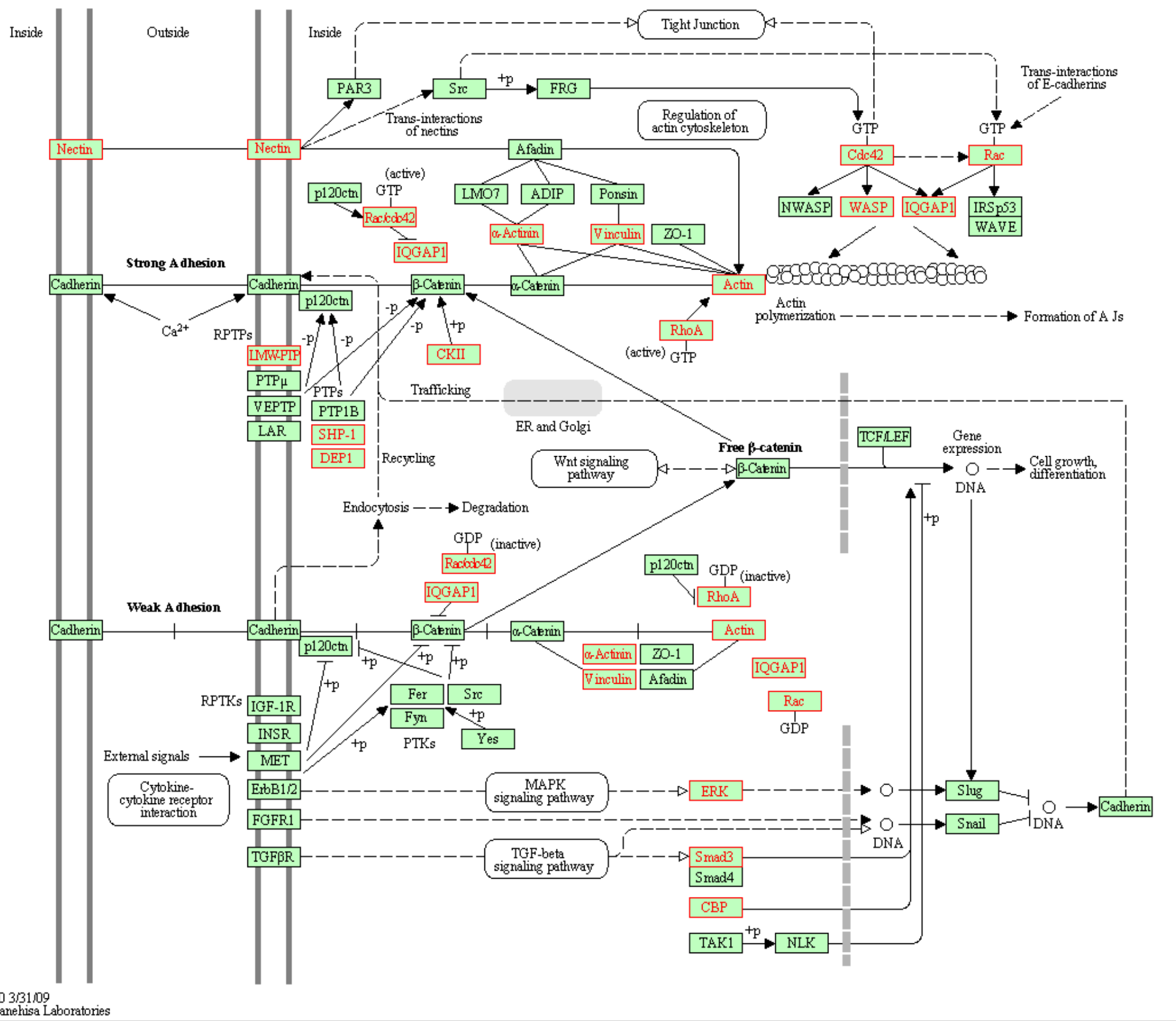




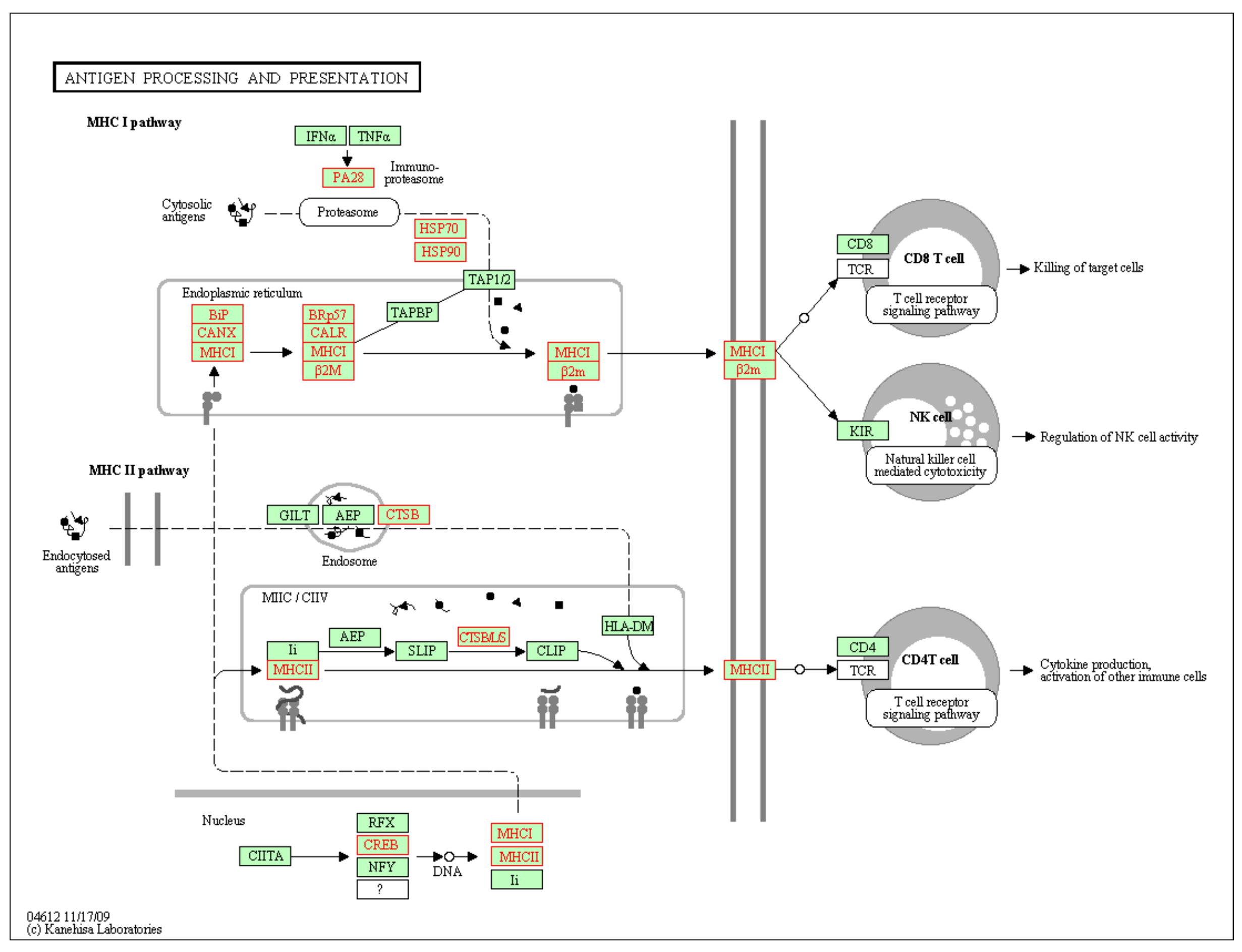

